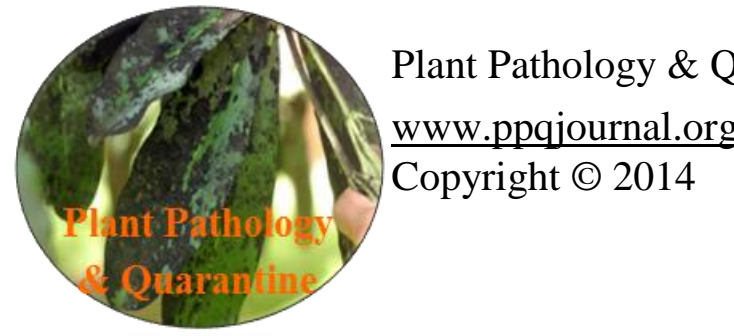

ISSN 2229-2217

\title{
Redisposition of species from the Guignardia sexual state of Phyllosticta Wulandari $\mathrm{NF}^{1,2^{*}}$, Bhat $\mathrm{DJ}^{3}$, and To-anun $\mathrm{C}^{1^{*}}$
}

\footnotetext{
${ }^{1}$ Department of Entomology and Plant Pathology, Faculty of Agriculture, Chiang Mai University, Chiang Mai, Thailand. ${ }^{2}$ Microbiology Division, Research Centre for Biology, Indonesian Institute of Sciences (LIPI), Cibinong Science Centre, Cibinong, Indonesia.

${ }^{3}$ Formerly, Department of Botany, Goa University, Goa-403 206, India
}

Wulandari NF, Bhat DJ and To-anun C. 2014 - Redisposition of species from the Guignardia sexual state of Phyllosticta. Plant Pathology \& Quarantine 4(1), 45-85, Doi 10.5943/ppq/4/1/6.

\begin{abstract}
Several species named in the genus "Guignardia" have been transferred to other genera before the commencement of this study. Two families and genera to which species are transferred are Botryosphaeriaceae (Botryosphaeria, Vestergrenia, Neodeightonia) and Hyphonectriaceae (Hyponectria). In this paper, new combinations reported include Botryosphaeria cocöes (Petch) Wulandari, comb. nov., Vestergrenia atropurpurea (Chardón) Wulandari, comb. nov., V. dinochloae (Rehm) Wulandari, comb. nov., V. tetrazygiae (Stevens) Wulandari, comb. nov., while six taxa are synonymized with known species of Phyllosticta, viz. Phyllosticta effusa (Rehm) Sacc.[(= Botryosphaeria obtusae (Schw.) Shoemaker], Phyllosticta sophorae Kantshaveli [= Botryosphaeria ribis Grossenbacher \& Duggar], Phyllosticta haydenii (Berk. \& M.A. Kurtis) Arx \& E. Müller [= Botryosphaeria zeae (Stout) von Arx \& E. Müller], Phyllosticta justiciae F. Stevens [= Vestergrenia justiciae (F. Stevens) Petr.], Phyllosticta manokwaria K.D. Hyde [= Neodeightonia palmicola J.K Liu, R. Phookamsak \& K. D. Hyde] and Phyllosticta rhamnii Reusser [= Hyponectria cf. buxi (DC) Sacc.]. In this paper, identification of "Guignardia" species is based on morphological characteristics. A large number of taxa labeled as "Guignardia" in literature, could not be loaned from various herbaria, or are lost or untraceable are listed as 'doubtful species' in view of insufficient supportive taxonomic data. Fresh collections, cultures and molecular sequence data are needed to clarify the phylogeny and taxonomy of cryptic species.
\end{abstract}

Key words - Dothideomycetes - Guignardia - plant diseases - saprobes - taxonomy

\section{Introduction}

In a recent work, 34 species in the genus Phyllosticta are accepted (Wulandari et al. 2013). The sexual state of Phyllosticta, "Guignardia", has previously been studied by several authors (Van der Aa 1973, Punithalingham 1974, Sivanesan 1984, Hyde 1995, Okane et al. 2001, Van der Aa \& Vanev 2002, Okane et al. 2003, Motohashi et al. 2008, Wulandari et al. 2009, Motohashi et al. 2010). The species of Guignardia have earlier been synonymized in genera such as Botryoshaeria, (Yamamoto 1961) and Glomerella (Arx \& Müller 1954). Presently, species of Guignardia are synonymized with 24 different genera. These include, Apioplagiostoma, Laestadia, Hyphonectria (Hyponectriaceae: Incertia sedis), Glomerella (Phyllachoraceae?: Phyllachorales), Apiosporopsis 
(Sordariales), Polystigma., Obryzum, Discosphaerina, Physalospora, Gelatinopsis, Lichenosticta, Didymella (Dothideales: Incertae Sedis), Plectosphaera, Verrucaria, Haloguignardia, Phyllachora (Phyllachoraceae: Phyllachorales), Botryosphaeria (Botryosphaeriaceae: Dothideales), Diaporthe (Diaporthales), Mycosphaerella (Mycosphaerellaceae: Dothideales), Rosselliniella, Puiggarina, Tellogalla, Catapyrenium, Isothea, Plectosphaera, Valsella (Valsaceae: Diaporthales) and Turgidosculum (Index Fungorum-http://www.indexfungorum.org/; accessed December 2012). This is primarily because this genus had a wide inclusiveness and any fungus with unicellular ascospores, any type of asci and simple ascomata were placed in this genus.

In this paper, we dealt with 304 species of sexual Phyllosticta ("Guignardia") and transferred several of them to appropriate species, but a large number are listed as doubtful species in view of insufficient supportive data. The aim of this paper is to redescribe some of the valid species which otherwise were not listed as "Guignardia" and to list those labeled as "Guignardia" but unable to be loaned, lost, traceable or doubtful species.

\section{Materials \& Methods}

Holotype specimen in the name of "Guignardia" were loaned from various International herbaria worldwide. Morphological examinations were done following the procedure described by Wulandari et al. (2010). Melzer's solution was used to identify the J-and J+ for unitunicate asci. All line drawings and photo-plates are prepared by the first author. Annotations were made for the loaned holotype specimen.

In Section I which includes all redisposed taxa, for each species, taxonomic nomenclature with citation, synonymy, MycoBank number (for new combinations only), species diagnosis, details of examined specimen and brief notes are given. In Section II which accommodates doubtful, untraceable, missing and invalid species of "Guignardia", details on taxonomy, citation, synonymy, herbarium, present status and needful notes are provided in tabular form. Wherever possible, descriptions are supported with photo-plates and line drawings of fungi.

\section{Results}

In this study of 304 taxa of sexual Phyllosticta ("Guignardia"), 14 species are transferred to 4 genera in 3 families and described in detail. The order and families to which species transferred include Botryosphaeriaceae (Botryosphaeria spp., Vestergrenia spp., Neodeightonia sp.) in Botryosphaeriales and Hyphonectriaceae (Hyponectria sp.). Of the remaining, 107 species are considered doubtful, lost or untraceable. The rest 183, so called "Guignardia" species, found in literature including Index Fungorum-http://www.indexfungorum.org/ could not be loaned during the time frame of the study. These 290 species of "Guignardia" are tabulated in Table 2 with details sourced from literature. It is hoped that the facts provided here will encourage and facilitate future workers to recollect fresh specimens and undertake further morpho-molecular studies.

\section{Taxonomy:}

\section{Redisposition of species of Guignardia}

Section 1: Transfered species

Botryosphaeriales C.L. Schoch, Crous \& Shoemaker, in Schoch, Shoemaker, Seifert, Hambleton, Spatafora \& Crous, Mycologia 98(6): 1050 (2007) [2006]

Botryosphaeriales presently comprises three families, Botryosphaeriaceae (29 genera), Phyllostictaceae (5 genera), and Planistromataceae (2 genera) (Liu et al. 2012, Hyde et al. 2013, Monkai et al. 2013).

Botryosphaeriaceae Ces. \& De Not., Comm. Soc. crittog. Ital. 1(4): 211 (1863)

Botryosphaeria Theiss. \& Syd. [as 'Botryosphaeriacae'], Annls mycol. 16(1/2): 16 (1918)

Botryosphaeria cocöes (Petch) Wulandari, comb. nov.

(Fig. 1a-g)

Index Fungorum: IF550638

= Guignardia cocöes (Petch) K.D. Hyde (1995); MycoBank: MB 447509

$\equiv$ Desmotascus cocöes Petch [as 'cocoes'] 1922 
Pathogenic causing leaf spots. Leaf spots brown, necrotic, with concentric rings of blackened dots. Ascomata developing under raised, carbonaceous, slightly darkened areas, occasionally erumpent and cracking the host surface, mostly solitary, in vertical section 250-300 $\mu \mathrm{m}$ diam., subglobose, immersed beneath the host cuticle, ostiolate. Peridium up to $25 \mu \mathrm{m}$ wide, composed of a few layers of brown-walled angular cells. Pseudoparaphyses not observed. Asci 75$125 \times 20-25 \mu \mathrm{m}, 8$-spored, clavate, pedicellate, bitunicate, fissitunicate, apically rounded, with an ocular chamber. Ascospores 23-26.5 × 9-10 $\mu \mathrm{m}, 2-3$-seriate irregularly ellipsoidal, 1-celled, hyaline, with apical button-like germ pores and remnants of mucilage on the surface.

Material examined - SOLOMON ISLANDS, on leaves of Cocos nucifera, June1917, Petch (K, from holotype of Guignardia cocöes).

Notes - This species is not a Guignardia but a species of Botryosphaeria. It is different from other species in the genus Botryosphaeria by ornamented, thick-walled ascospores. Known distribution of the fungus is in Solomon Islands (Sivanesan 1984, Hyde 1995).

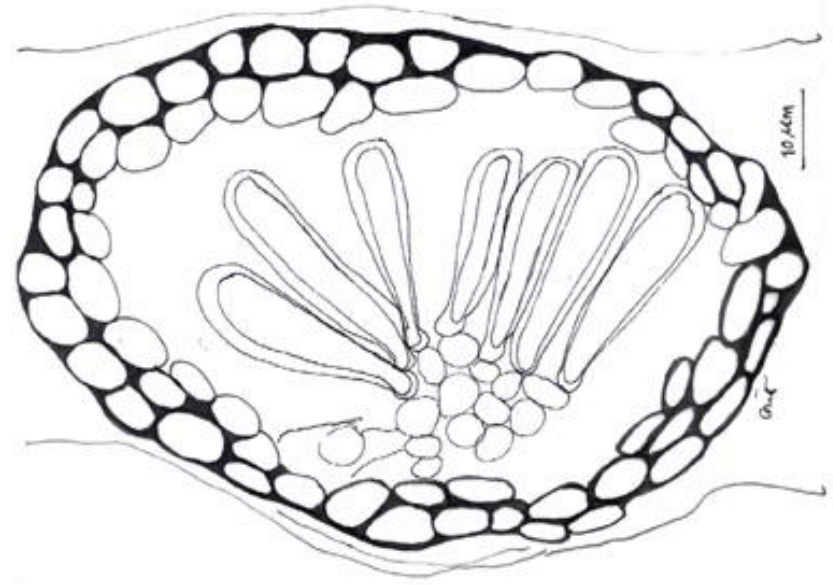

a
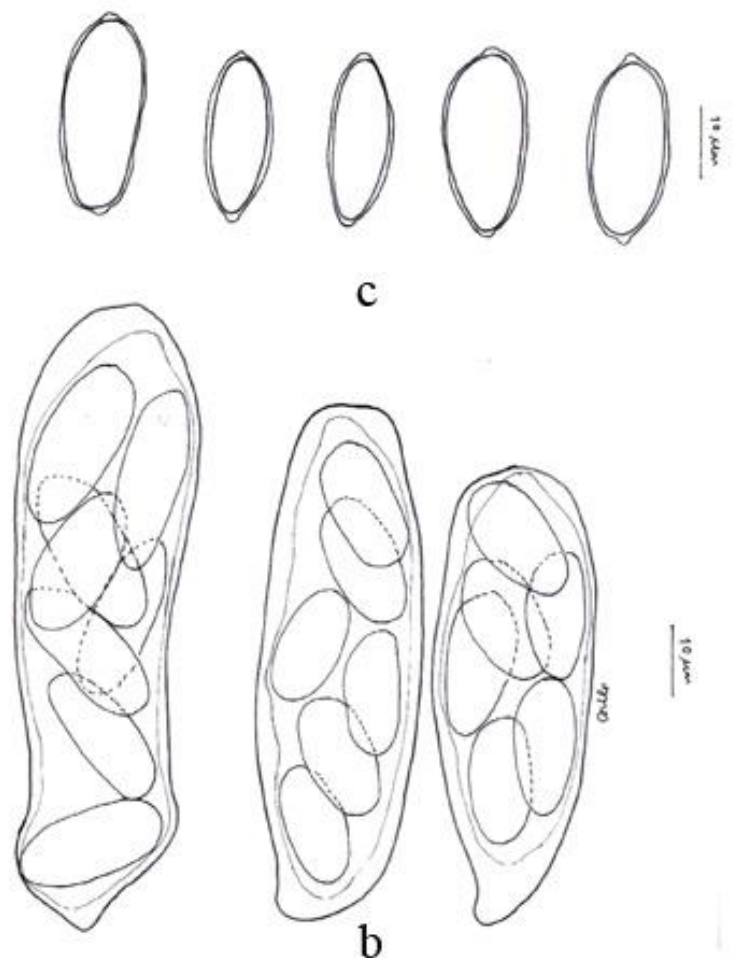

Figs 1a-c - Botryosphaeria cocöes (K, from holotype of Guignardia cocöes). a, Peridium comprising one strata of 2-4 cells textura angularis with thickened brown walls. b, Asci. c, Ascospores. - Scale bars a-c $=10 \mu \mathrm{m}$. 
Botryosphaeria obtusae (Schw.) Shoemaker, Canadian Journal of Botany 42: 1298 (1964)

= Guignardia effusa (Rehm) Sacc., Syll. fung. (Abellini) 24(2): 784 (1928) MycoBank: MB 213834

三Laestadia effusa Rehm (1915), Annls mycol. 13(1): 4 (1915)

Ascomata 150-200 $\mu \mathrm{m}$ diam., 155-200 $\mu \mathrm{m}$ high, scattered, solitary, dark brown to black, globose to subglobose, immersed to semi-immersed in plant tissues. Peridium 15-20 $\mu \mathrm{m}$ wide, one strata of 2-4 layers of cells textura angularis with thickened brown walls around ostiole. Asci 70$145 \times 11-25 \mu \mathrm{m}(\bar{x}=100 \times 20 \mu \mathrm{m}, \mathrm{n}=20), 8$-spored, bitunicate, cylindrical to cylindric-clavate, rounded at the apex where the diam. is 10-20 $\mu \mathrm{m}$, tapering gradually to $15-26 \times 5-7 \mu \mathrm{m}$ long pedicel attached to the basal peridium. Ascospores $20-30 \times 7-10 \mu \mathrm{m}(\bar{x}=25 \times 10 \mu \mathrm{m}, \mathrm{n}=20)$, irregularly biseriate, fusoid, wider around the mid region, hyaline to greenish, 1-celled, guttulate, smooth-walled, without mucilaginous sheath at the ends.

Material examined - UK, London, on stems of Milium effusum, 31 January 1914 (F 10850, from holotype of Guignardia effusa).

Notes - Botryosphaeria obtusae, conforms its identity, as the morphological characters are similar with the description given by Sivanesan (1984). The asexual state is Diplodia seriata (Sivanesan 1984). Botryosphaeria obtusae is responsible for one of important plant diseases in Tunisia, olive tree branch dieback (Chattaoui et al. 2012). ITS 1 and ITS 4 were used to identify this species from dieback and olive root rot symptoms in Tunisia (Chattaoui et al. 2012).
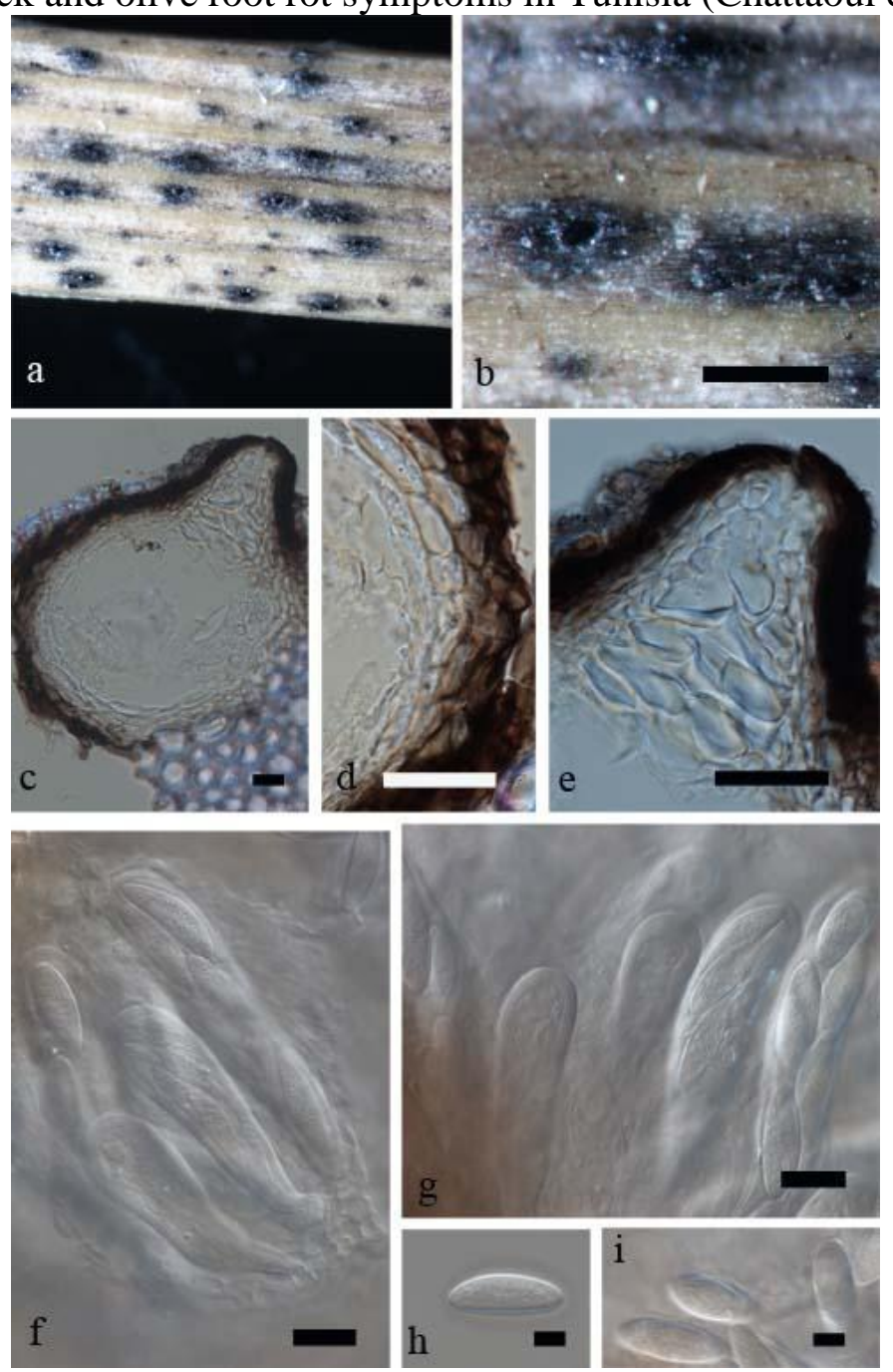

Figs 2a-i - Botryosphaeria obtusae (FS 10850, from holotype of Guignardia effusa). a, b Ascomata on host surface. c-e Peridium comprising one strata of 2-4 cells textura angularis with thickened brown walls. f, g Asci. h, i Ascospores. - Scale bars b, c $=100 \mu \mathrm{m}, \mathrm{d}=40 \mu \mathrm{m}, \mathrm{e}, \mathrm{f}=10$ $\mu \mathrm{m}, \mathrm{g}=20 \mu \mathrm{m}$. 


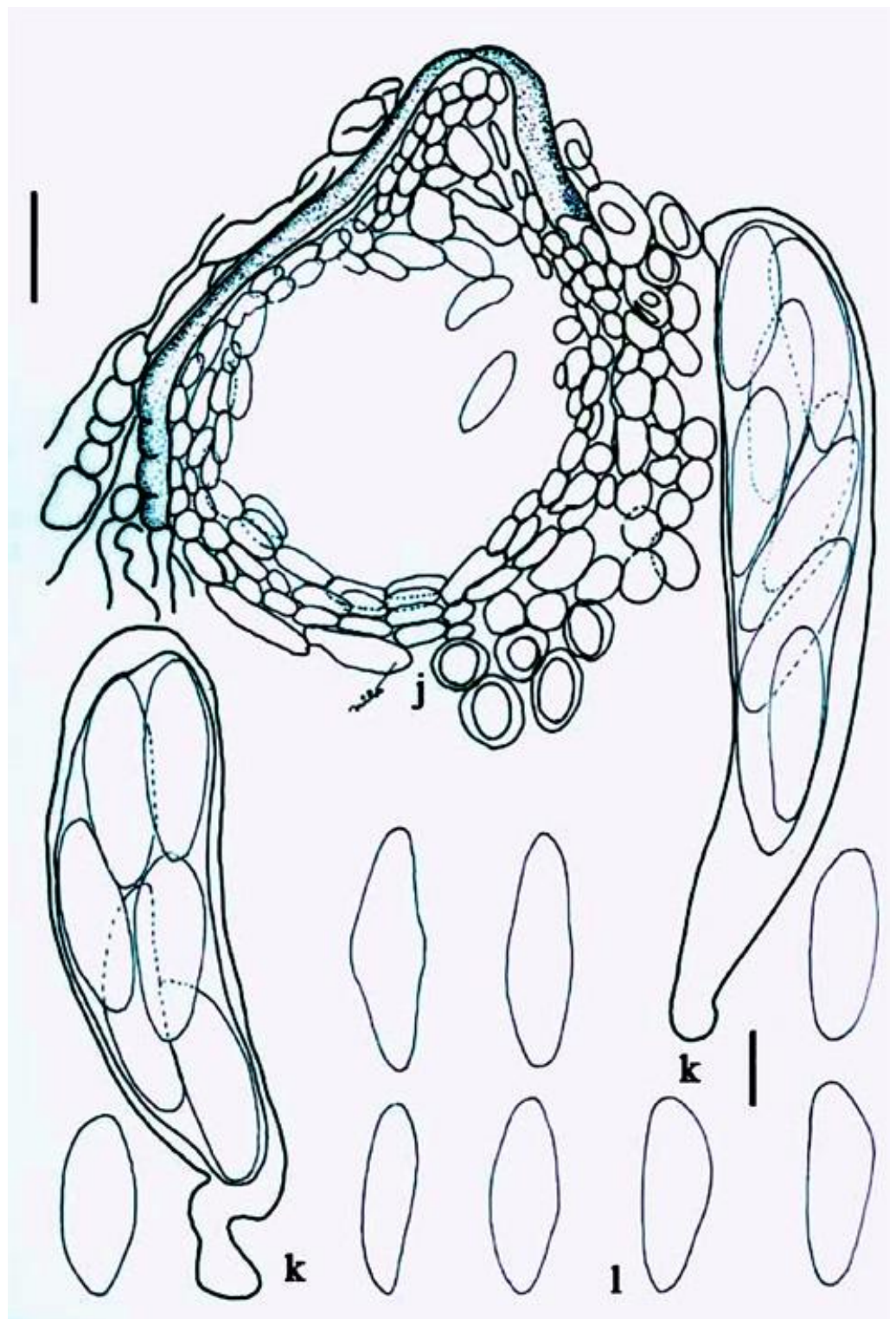

Figs 2j-l - Botryosphaeria obtusae (F 10850, from holotype of Guignardia effusa) line drawing. j, Peridium comprising one strata of 2-4 cells textura angularis with thickened brown walls. $\mathrm{k}$, Asci. 1, Ascospores. - Scale bars $=25 \mu \mathrm{m}$.

Botryosphaeria ribis Grossenbacher \& Duggar, Technical Bulletin New York Agricultural Experiment Station 18: 128 (1911)

(Fig. 3a-g)

= Guignardia sophorae Kantshaveli, Morbi plantarum 17: 84 (1928) MycoBank: 278408

Ascomata 125-200 $\mu \mathrm{m}$ diam., 125-205 $\mu \mathrm{m}$ high, on surface of the leaves, black, globose to subglobose, immersed to semi-immersed in plant tissues. Peridium 38-63 $\mu \mathrm{m}$ wide, one strata of 24 layers of cells textura angularis, with thickened brown walls around ostiole. Asci 55-90 × 13-23 $\mu \mathrm{m}(\bar{x}=71 \times 19 \mu \mathrm{m}, \mathrm{n}=20), 8$-spored, bitunicate, cylindrical to cylindric-clavate, rounded at the apex where the diam. is $13-20 \mu \mathrm{m}$, tapering gradually to a $8-23 \times 8-15 \mu \mathrm{m}$ long pedicel attached to the basal peridium. Ascospores 18-25 $\times 5-10 \mu \mathrm{m}(\bar{x}=21 \times 7 \mu \mathrm{m}, \mathrm{n}=20)$, uniseriate to biseriate, ellipsoidal, widest $2 / 5$ near the apex (obtrullate), hyaline to greenish, 1-celled, coarse-guttulate, smooth-walled, without mucilaginous sheath at the ends.

Material examined - GEORGIA, Tiflis on stems of Sophora japonica (Fabaceae), 25 October 1936, Kantshaveli, V. L. Komarov Botanical Institute. Prof. Popov Street 2. Saint Petersburg 197376, Russia (LE 34626, from holotype of Guignardia sophorae). 
Notes - Morphologically the specimen labeled as Guignardia sophorae is similar to Botrysphaeria ribis (Sivanesan 1984) and hence the transfer was done. The asexual state is Fusicoccum ribis (Macedo \& Baretto 2008). Three genes sequenced (ITS, EF and B-tubulin) showed two distinct phylogenetic assemblages within two asexual morphs (Fusicoccum and Diplodia) (Slippers et al. 2004).

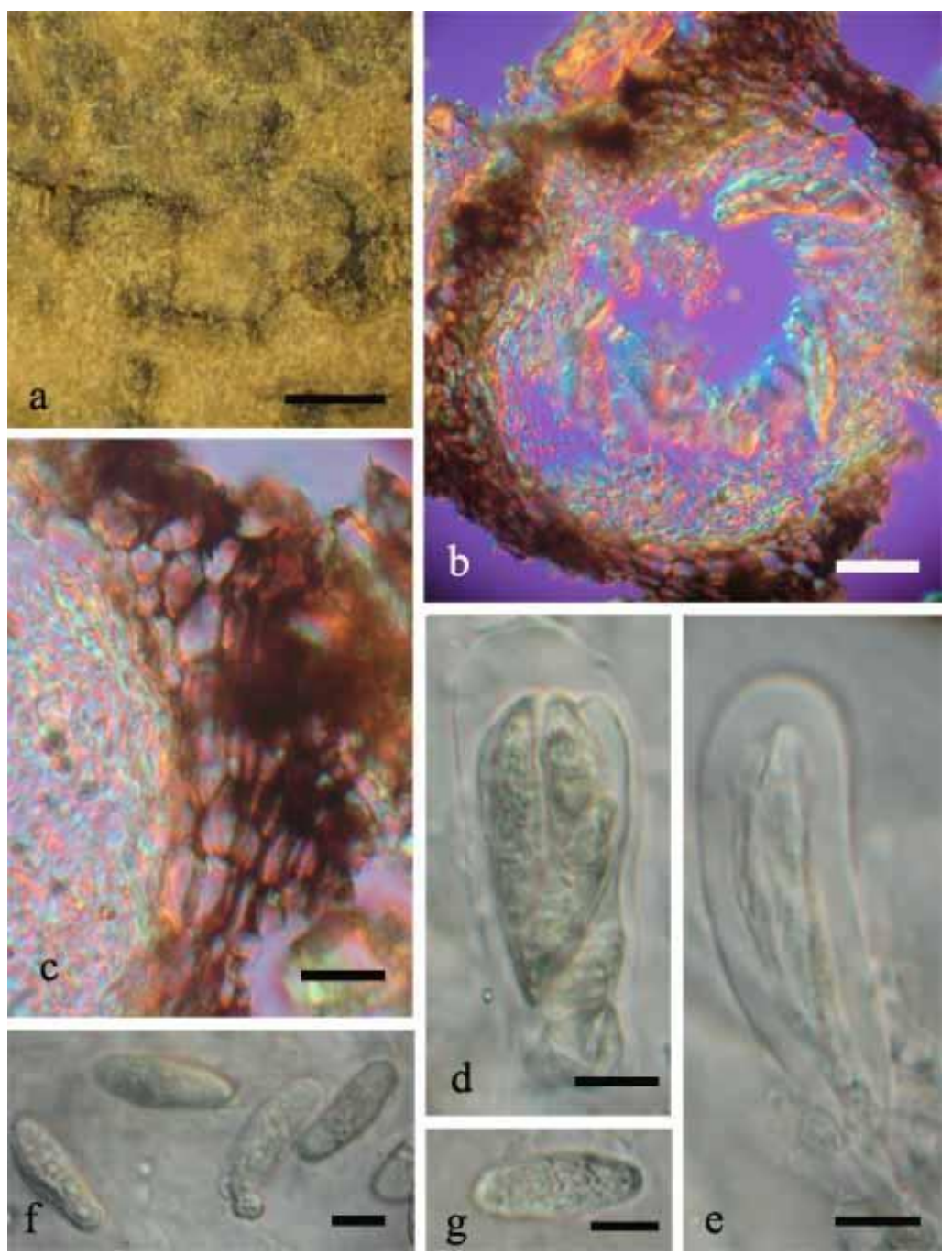

Figs 3a-g - Botryosphaeria ribis (LE 34626, from holotype of Guignardia sophorae). a, Ascomata on host surface. b, c Peridium comprising one strata of 2-3 cells textura angularis with thickened brown walls. d, Asci. e, Immature asci. f, g Ascospores. - Scale bars a $=200 \mu \mathrm{m}, \mathrm{b}, \mathrm{c}=40 \mu \mathrm{m}, \mathrm{d}$, g $=10 \mu \mathrm{m}$.

Botryosphaeria zeae (Stout) von Arx \& E. Müller,

(Fig. 4a-i)

Beitr. Krypt.- fl. Schweiz 11: 40 (1954)

= Guignardia haydenii (Berk. \& M.A. Curtis) Arx \& E. Mull. (1954) MycoBank: MB 344112

$\equiv$ Dothidea haydenii Berk. \& M.A. Curtis, Grevillea 4 (no. 31): 104 (1876)

Ascomata in groups and connected to each other, individually $28-80 \mu \mathrm{m}$ diam., $90-200 \mu \mathrm{m}$ high, black, globose to rectangular, immersed in plant tissue; in vertical section subglobose, with an indistinct ostiole. Peridium 13-67 $\mu \mathrm{m}$ diam. in the upper region, 11-13 $\mu \mathrm{m}$ in the base, comprising two strata of thickened black-walled cells textura angularis, with inner wall layer cells paler and vertical. Asci 41-66 × 12-16 $\mu \mathrm{m}, 6-8$-spored, bitunicate, with ocular chamber, attached to the basal peridium, cylindrical to slightly clavate, rounded at the apex where diam. is $7-10 \mu \mathrm{m}$, tapering gradually a to $15-26 \times 5-7 \mu \mathrm{m}$ short pedicel attached to the basal peridium. Ascospores $14-17 \times 4-$ 
$6 \mu \mathrm{m}$, biseriate, overlapping, ellipsoidal to cylindrical, slightly fusiform, hyaline, 1-celled, guttulate, smooth-walled, without a mucilaginous sheath.

Material examined - Near Fargo, N. Dak., on stems of Aster paniculatus (Asteraceae), 5 June 1915, Brenckle and Stevens, Fungi Dakotensis Brenckle (BPI 845200, from isotype of Guignardia haydenii).

Notes - Morphology of Guignardia haydenii is similar to Botryosphaeria zeae (Sivanesan 1984) and therefore this transfer was done. So far, no molecular study has been done for this taxon.

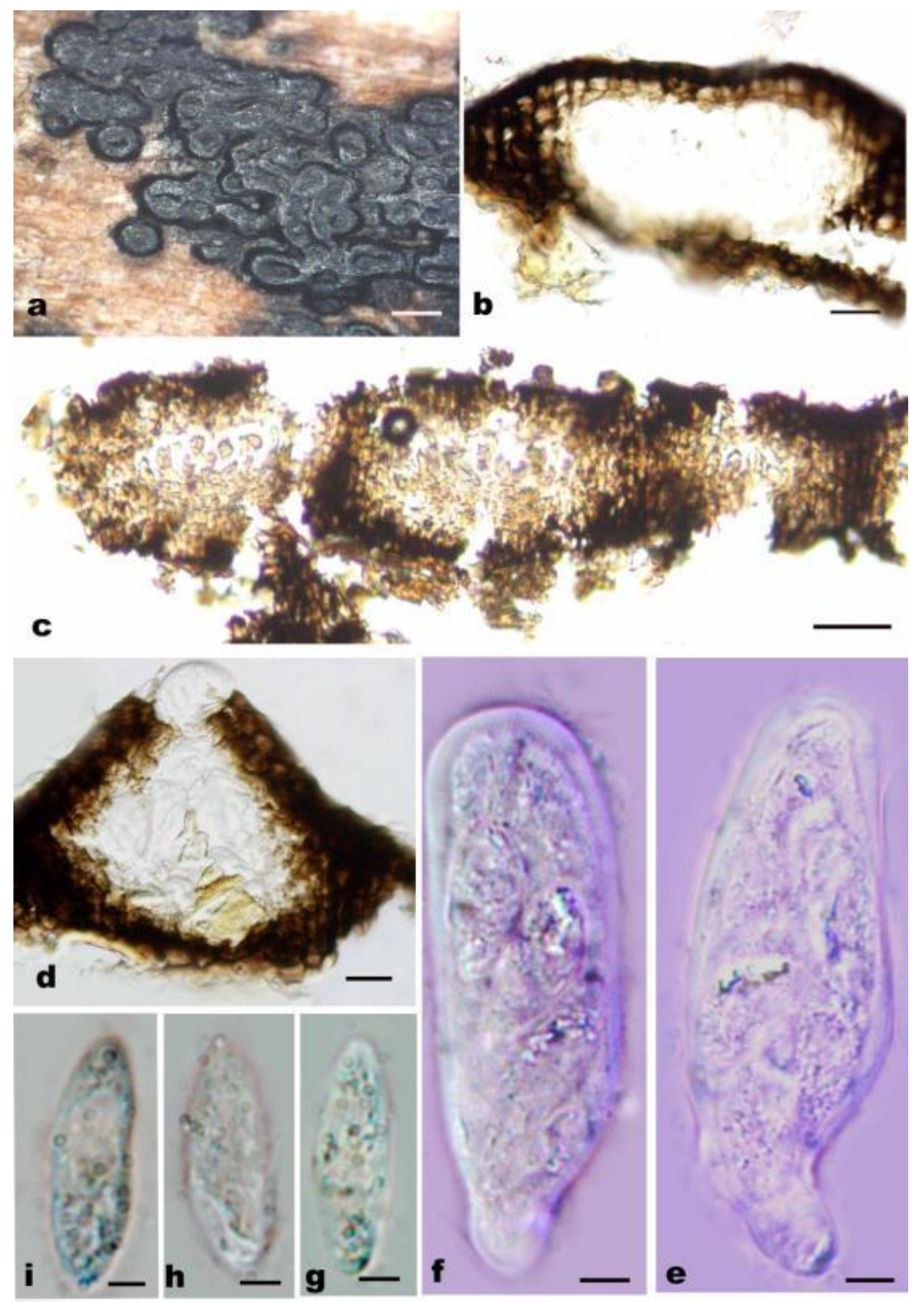

Figs 4a-i - Botryosphaeria zeae (BPI 845200, from isotype of Guignardia haydenii). a, Ascomata on host surface. b-d. Peridium comprising one strata of 2-4 cells of textura angularis with thickened brown walls. e, f Asci. g-i. Ascospores. - Scale bars $\mathrm{a}=100 \mu \mathrm{m}, \mathrm{b}, \mathrm{c}=100 \mu \mathrm{m}, \mathrm{d}=40$ $\mu \mathrm{m}, \mathrm{e}, \mathrm{f}=10 \mu \mathrm{m}, \mathrm{g}-\mathrm{i}=10 \mu \mathrm{m}$.

Vestergrenia Rehm, Hedwigia 40: 100 (1901)

Vestergrenia atropurpurea (Chardon) Wulandari, comb. nov.

(Fig. 5a-g)

Index Fungorum: IF550639

= Guignardia atropurpurea Chardón, Mycologia 32(2): 177 (1940) MycoBank: MB 286749 
Parasitic leaves with irregular leaf spots, with ascomata visible on the surface as numerous black dots. Ascomata 200-250 $\mu \mathrm{m}$ diam., 175-250 $\mu \mathrm{m}$ high, epiphyllous, black, pseudothecium, globose to subglobose, immersed in plant tissues, coriaceous, solitary to clustered, ostiolate, with ostioles as a black dots in the centre. Peridium 12.5-37.5 $\mu \mathrm{m}$ wide, comprising one strata of 2-3 layers of cells textura angularis with thickened brown walls. Pseudoparaphyses not observed. Asci 74-154 × 20-26 $\mu \mathrm{m}(\bar{x}=118 \times 22 \mu \mathrm{m}, \mathrm{n}=20), 8$-spored, bitunicate, without a distinct ocular chamber subclavate to cylindrical, rounded at the apex where the diam. is $12-22 \mu \mathrm{m}$, tapering gradually to a 16-92 $\times 4-6 \mu \mathrm{m}$ long pedicel attached to the basal peridium. Ascospores 14-18 $\times 6-$ $10 \mu \mathrm{m}(\bar{x}=16 \times 8 \mu \mathrm{m}, \mathrm{n}=20)$, biseriate, ellipsoidal, hyaline to greenish, 1-celled, coarse-guttulate, smooth-walled, without a mucilaginous sheath.

Material examined - BRAZIL, Viçosa, on dead leaves of Miconia sp. (Melastomataceae), 4 January 1933, Fungus de Minas Geraes, Müller 443 (BPI 598138, from holotype of Guignardia atropurpurea).

Notes - With its carbonaceous, large ascomata and oblong ascospores, Guignardia atropurpurea is morphologically similar to Vestergrenia (Liu et al. 2012). However, this does not conform to other described species in the genus and therefore new combination is proposed.

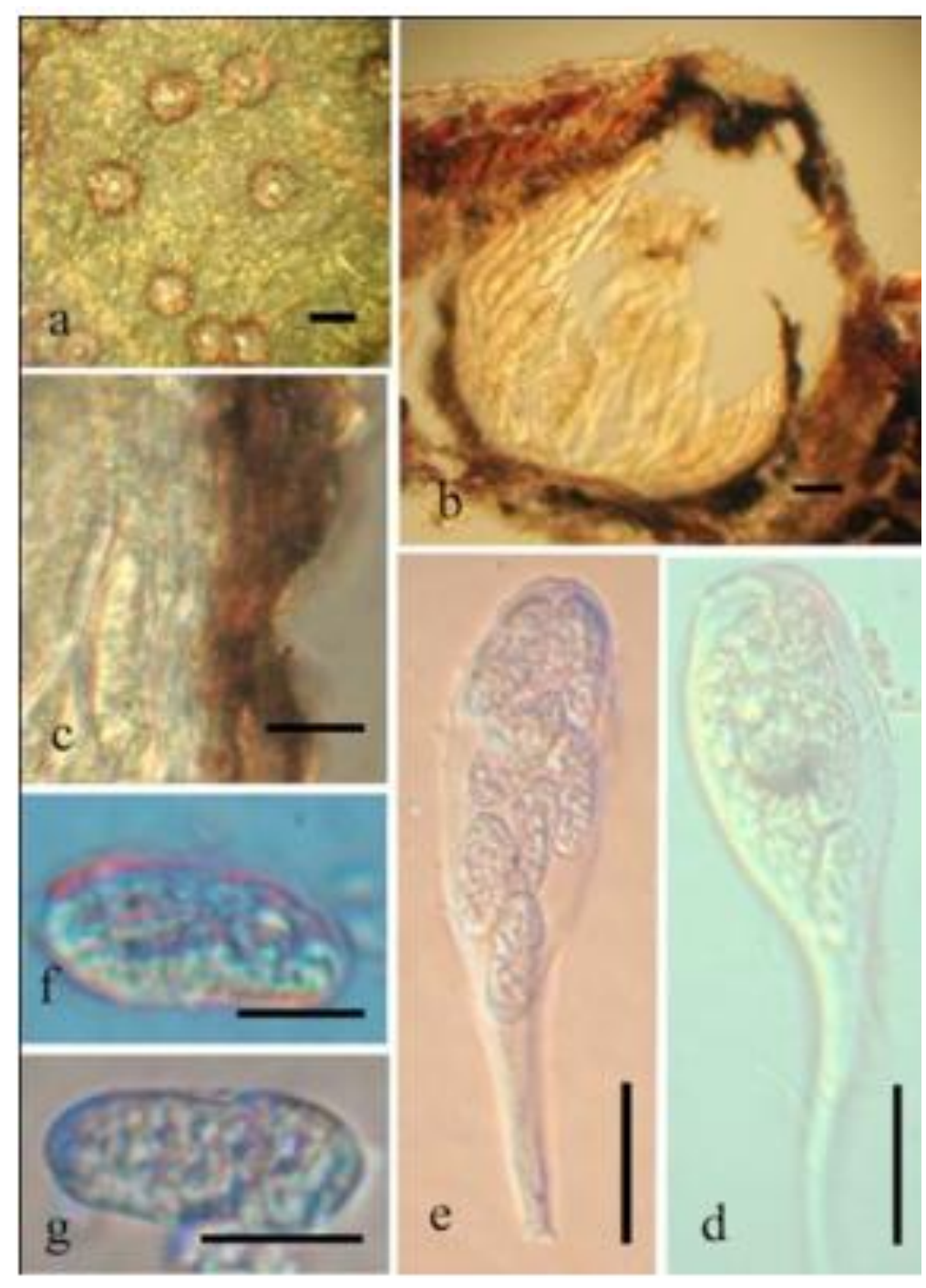

Fig. 5a-g - Vestergrenia atropurpurea (BPI 598138, from holotype of Guignardia atropurpurea) a.Ascomata on the host surface. b-c. Peridium comprising one strata comprising 2-3 layers of cells of textura angularis with thickened brown walls. d-e. Asci. f-g. Ascospores. Scale bars a $=200 \mu \mathrm{m}$, b-c $=15 \mu \mathrm{m}, \mathrm{d}-\mathrm{e}=26 \mu \mathrm{m}, \mathrm{f}-\mathrm{g}=10 \mu \mathrm{m}$. 
Saprobic on leaf litter with irregular spots, with ascomata visible on leaf spots as black dots. Ascomata 200-250 $\mu \mathrm{m}$ diam., 190-200 $\mu \mathrm{m}$ high, pseudothecium, epiphyllous, black, globose to subglobose, immersed in plant tissues, coriaceous, solitary to clustered, ostiolate, with ostiole as a black dot in the centre. Peridium 35-40 $\mu \mathrm{m}$ wide, comprising one strata of 2-3 layers of cells textura angularis with thickened brown walls. Pseudoparaphyses not observed. Asci 60-110 $\times 15-$ $26 \mu \mathrm{m}(\bar{x}=90 \times 20 \mu \mathrm{m}, \mathrm{n}=20), 8$-spored, bitunicate, fissitunicate, pedicel attached to the basal peridium. Ascospores $14-18 \times 6-10 \mu \mathrm{m}(\bar{x}=16 \times 8 \mu \mathrm{m}, \mathrm{n}=20)$, biseriate, ellipsoidal, hyaline to greenish, 1-celled, coarse-guttulate, smooth-walled, without a mucilaginous sheath.

Material examined - PHILIPPINES, Mount Maquiling, near Los Banos province, Laguna, on Dinochloa sp. (Poaceae). December 1913, C.F. Baker (F 81683, from holotype of Guignardia dinochloae; F 81684; F 81684).

Notes - This specimen differs from other Vestergrenia by presence of periphyses on the ostiolar neck of ascomata. The ascomata is fully immersed in the host and not visible on the surface of the leaves as in other species of Vestergrenia.
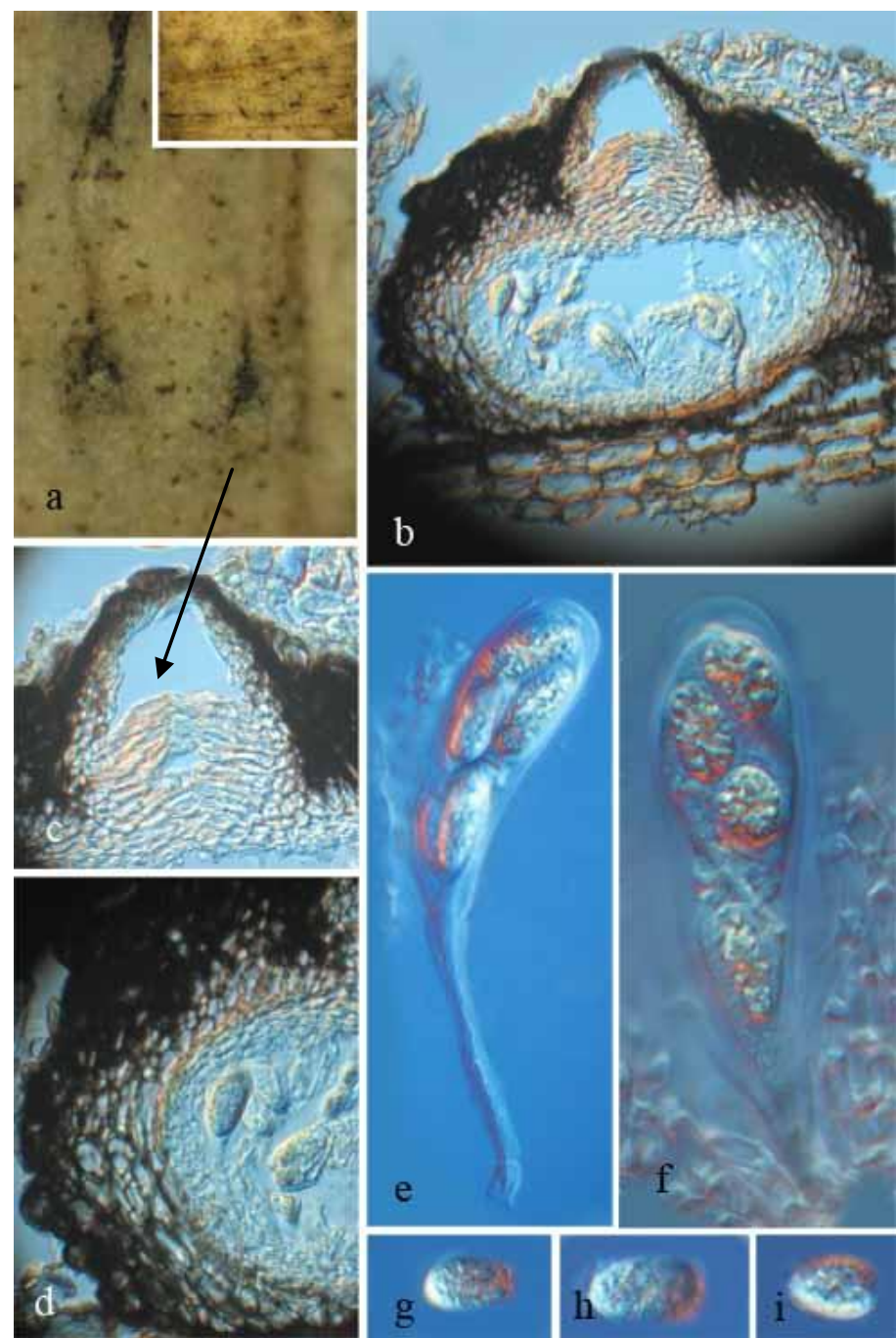

Figs 6a-g - Vestergrenia dinochloae (F 81683, from holotype of Guignardia dinochloae) a, Ascomata on the host surface. b, d. Peridium comprising one strata of 2-3 layers of cells textura angularis with thickened brown walls. c, Ascomata neck with periphyses (arrow). d-f. Asci (fissitunicate). $\mathrm{g}$, Ascospores. - Scale bars $\mathrm{a}=200 \mu \mathrm{m}, \mathrm{b}, \mathrm{c}=50 \mu \mathrm{m}, \mathrm{d}, \mathrm{e}=10 \mu \mathrm{m}, \mathrm{f}=26 \mu \mathrm{m}, \mathrm{g}=$ $10 \mu \mathrm{m}$. 
Ascomata 200-250 $\mu \mathrm{m}$ diam., 250-288 $\mu \mathrm{m}$ high, on the surface of the leaves, pseudothecium, black, globose to subglobose, semi-immersed in tanned plant tissues, hard, solitary, ostiolate, with ostioles as a black dots in the centre. Peridium 38-50 $\mu \mathrm{m}$ wide, comprising one strata of 2-3 layers of cells of textura globularis with thickened brown walls. Pseudoparaphyses not observed. Asci 73-125 × 16-26 $\mu \mathrm{m}(\bar{x}=92 \times 22 \mu \mathrm{m}, \mathrm{n}=20)$, 8-spored, bitunicate, fissitunicate, with an ocular chamber 3-6 $\mu \mathrm{m}$ high, broadly cylindrical to cylindro-clavate, rounded at the apex where the diam. is $13-18 \mu \mathrm{m}$, tapering gradually to a $19-63 \times 4-5 \mu \mathrm{m}$ long pedicel attached to the basal peridium. Ascospores 9-26 $\times 6-13 \mu \mathrm{m}(\bar{x}=20 \times 9 \mu \mathrm{m}, \mathrm{n}=20)$, overlapping biseriate, ovoid, diamond-shaped, hyaline to greenish, 1-celled, coarse-guttulate, smooth-walled, with a mucilaginous sheath on both ends.

Material examined - PUERTO RICO, Maricao, on leaves of Justicia verticillaris (Acanthaceae). 28 August 1913, F.L. Stevens, Private Herbarium F.L. Stevens No. 806, University Illinios Herbarum No. 2839 (ILL 9738, from holotype of Guignardia justiciae); PUERTO RICO, El Yungue, on the leaves of Justicia verticillaris, 16 July 1915, F. L. Stevens, University Illinios Herbarum No. 8557 (ILL9737, from paratype of Guignardia justiciae).

Notes - This species differs from other Vestergrenia species, by unique peridium with cells of textura globularis and diamond-shaped ascospores. In others, the cells of the peridium are texura angularis and ascospores ellipsoidal (Liu et al. 2012)

Vestergrenia tetrazygiae (Stevens) Wulandari, comb. nov.

(Fig. 8a-i)

Index Fungorum: IF550641

= Guignardia tetrazygiae F. Stevens, Bot. Gaz. 69: 255 (1920) MycoBank: MB 528760

Ascostromata 150-188 $\mu \mathrm{m}$ diam., 150-200 $\mu \mathrm{m}$ high, on upper and lower leaf surface, pseudothecium, black, globose to sub-globose, immersed in plant tissues. Peridium 38-53 $\mu \mathrm{m}$ wide, in one strata 1-2 layers of cells textura angularis with thickened brown angular walls. Asci 63-150 $\times 25-38 \mu \mathrm{m}(\bar{x}=104 \times 31 \mu \mathrm{m}, \mathrm{n}=10), 8$-spored, bitunicate, cylindrical to cylindroclavate, rounded at the apex where the diam. is $18-25 \mu \mathrm{m}$, tapering gradually to a $25-75 \times 3-8 \mu \mathrm{m}$ long pedicel attached to the basal peridium. Ascospores 18-28 $\times 10-13 \mu \mathrm{m}(\bar{x}=24 \times 12 \mathrm{um}, \mathrm{n}=$ 20), biseriate, ellipsoidal, oblong, hyaline to greenish, 1-celled, coarse-guttulate, smooth-walled, without mucilaginous appendage at the ends.

Material examined - Puerto Rico, Vega Alga, on leaves of Tetrazygia eleagnoidea (Melastomataceae), Nov. 1913, F.L Stevens (BPI 844972, from holotype of Guignardia tetrazygiae).

Notes - With ascostromata growing on the veins of the leaves, asci with long pedicel, ellipsoidal to ovoid and guttulate ascospores with a thick perispore, Guignardia tetrazygiae is similar to Vestergrenia (Liu et al. 2012). However, its small-sized ascomata is unique and therefore the new combination is proposed.

Neodeightonia C. Booth, in Punithalingam, Mycol. Pap. 119: 17 (1970) [1969]

Neodeightonia palmicola J.K Liu, R. Phookamsak \& K.D. Hyde., Sydowia 62(2):261-276 (2010)

= Guignardia manokwaria K.D. Hyde, Sydowia 47(2): 191 (1995) MycoBank: MB 413137

(Fig. 9a-i)

Ascomata developing under slightly darkened area, occasionally erumpent and cracking the host surface, mostly solitary, in vertical section ca $200 \mu \mathrm{m}$ diam., $100 \mu \mathrm{m}$ high, conical, immersed beneath the host cuticle with an erumpent apex, base flattened, with a thin spreading stroma at the periphery of the ascoma. Peridium up to $30 \mu \mathrm{m}$ wide, thin below, composed of dark-brown-walled angular cells at the sides. At the periphery is a wedge of dark-brown angular cells, which extends as a thin line between adjacent ascomata. Pseudoparaphyses up to $4 \mu \mathrm{m}$ diam., hypha-like, 
filamentous, composed of short cylindrical cells, 8-10 ×4 $\mu \mathrm{m}$ diam. Asci 70-100 $\times 20-24 \mu \mathrm{m}$, 8spored, clavate, pedunculate, bitunicate, fissitunicate, apically rounded with an ocular chamber and faint ring. Ascospores 22-30 $\times 8-12 \mu \mathrm{m}, 2-3$-seriate, fusiform or fusiform-rhomboid, unicellular, hyaline, with apical button-like germ-pores and surrounded by a mucilaginous sheath with an irregular wavy outline.

Material examined - INDONESIA, Irian Jaya, Manokwari, on rachides of dead Gelubia sp., (Arecaceae) Mar. 1992, K.D. Hyde and N. Ragä (KDH 1206, from holotype of Guignardia manokwaria, BRIP 22749, phototype).

Notes - Similarities in morphology and molecular sequences data lead synonymising Guignardia manokwaria under Neodeightonia palmicola J.K Liu, R. Phookamsak and K.D. Hyde (Liu et al. 2011).
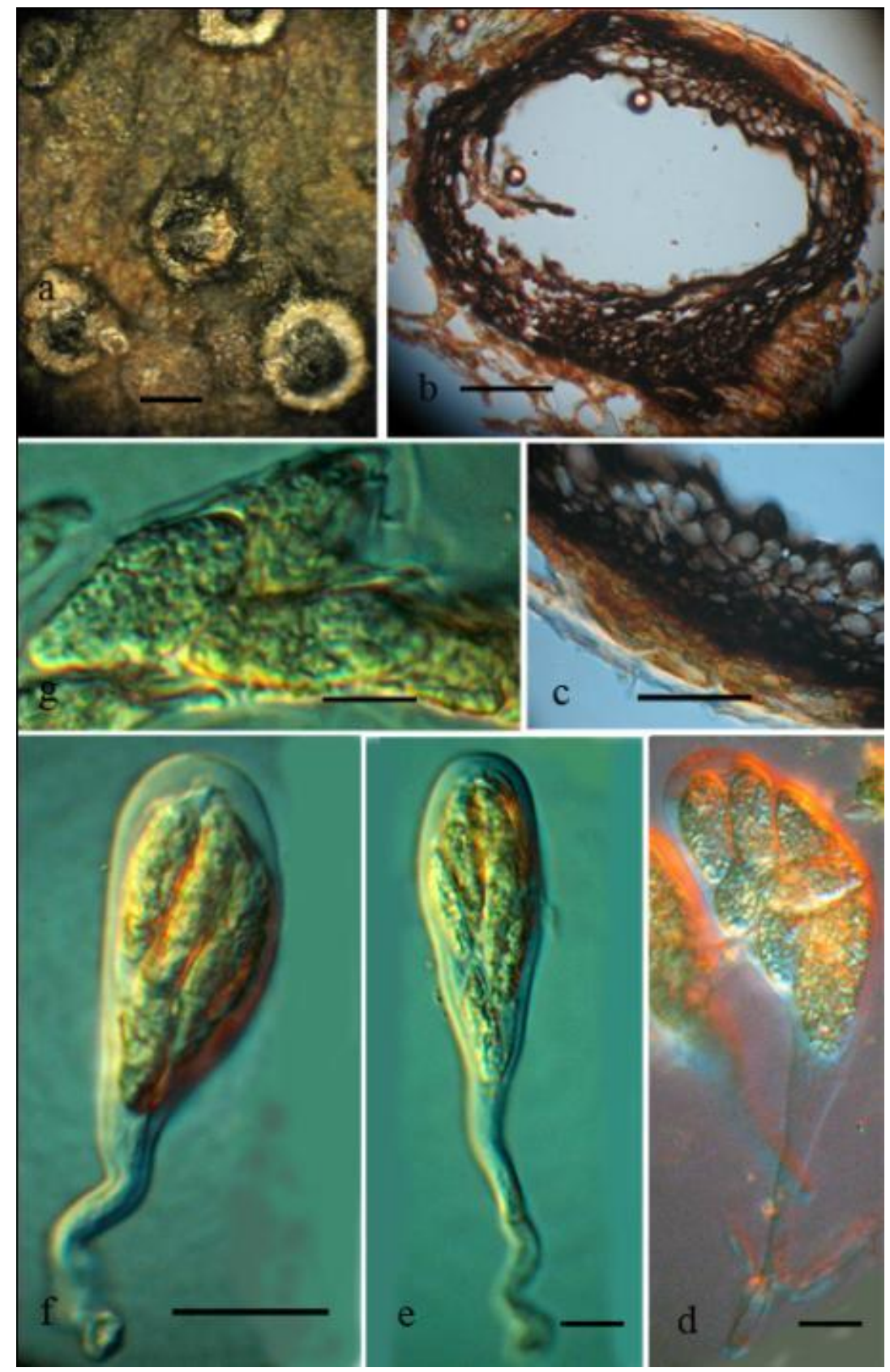

Fig. 7a-g - Vestergrenia justiciae (ILL 9738, from holotype of Guignardia justiciae) a, Ascomata on the host surface. b, c Peridium comprising one strata 2-3 layers of cells of textura angularis with thickened brown walls. d-f Asci. g, Ascospores. - Scale bars $\mathrm{a}=200 \mu \mathrm{m}, \mathrm{b}, \mathrm{c}=50 \mu \mathrm{m}, \mathrm{d}, \mathrm{e}=$ $10 \mu \mathrm{m}, \mathrm{f}=26 \mu \mathrm{m}, \mathrm{g}=10 \mu \mathrm{m}$. 
Hyponectriaceae Petr. (1923), as a family has accommodated 22 genera with 15 synonyms and 128 species (Kirk et al. 2008). Hyponectria Sacc. (1978), a genus of Hyponectriaceae, is wide spread on dead leaves and other substrates, and consists of 16 species (Index Fungorum; Kirk et al. 2008). Index fungorum lists 49 species or epithets. The affinities of all these remains uncertain, pending further molecular analyses. Physalospora Niessl. and Hyponectria Sacc., are distinguished by the size of ascomata and ascospores. The ascomata in Physalospora is up to $400 \mu \mathrm{m}$ diam. and ascospores range from 20 up to $30 \mu \mathrm{m}$, whereas in Hyponectria the ascomata is rarely up to $200 \mu \mathrm{m}$ diam. and ascospores length rarely extend over $20 \mu \mathrm{m}$. Both Physalospora and Hyponectria react with chitinoid (J+ or and J-) (Barr 1977).

Hyponectriaceae Petr., Annls mycol. 21(3/4): 305 (1923)
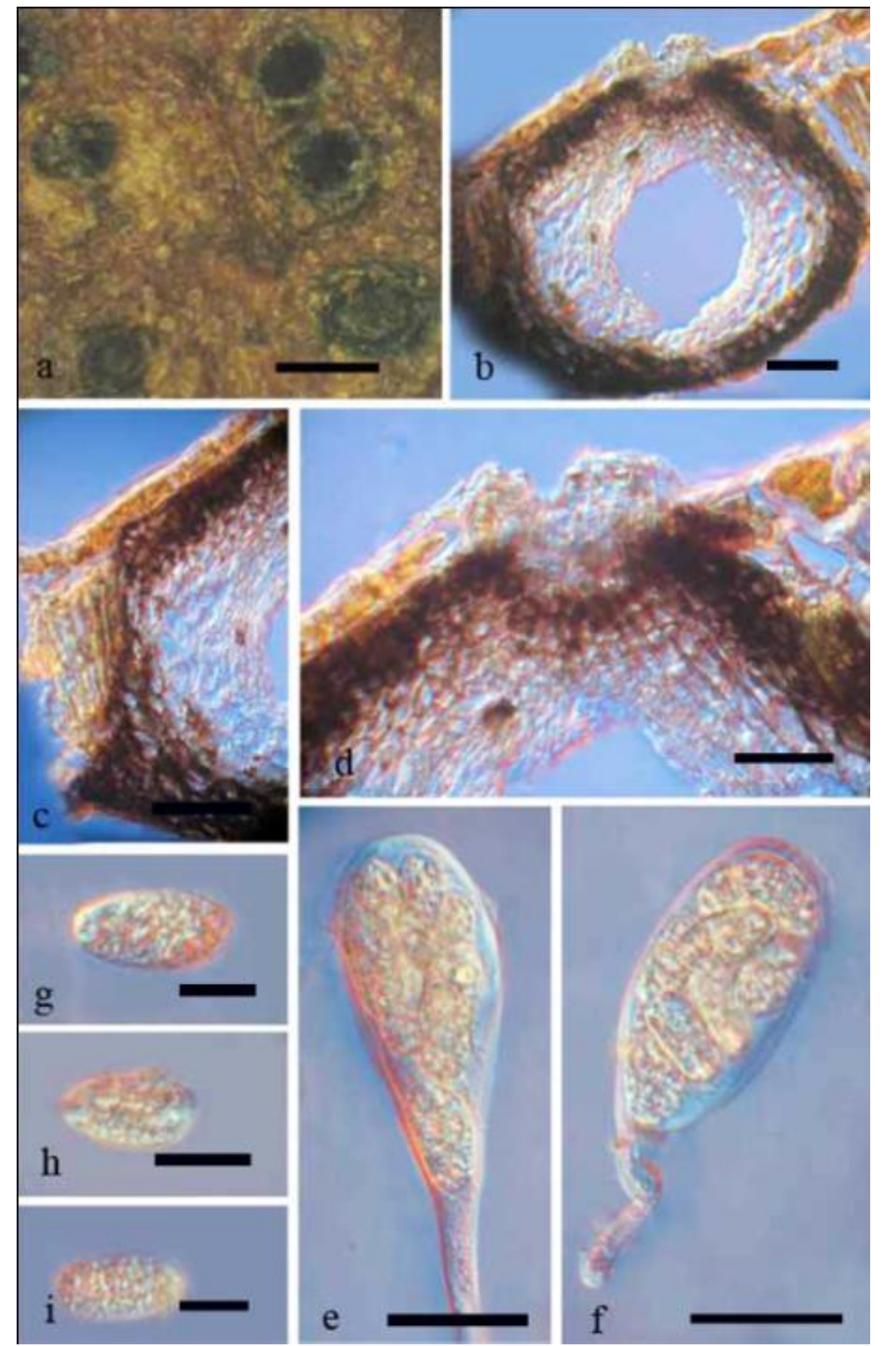

Figs 8a-i - Vestergrenia tetrazygiae (BPI 844972, from holotype of Guignardia tetrazygiae). a, b Ascomata on host surface. c, d Peridium comprising 1-2 cells of textura angularis with thickened angular brown walls. e, f Asci. g, i Ascospores clavate viewed in any plane. Scale bars $\mathrm{a}=200 \mu \mathrm{m}$, $\mathrm{b}-\mathrm{d}=40 \mu \mathrm{m}, \mathrm{e}-\mathrm{f}=30 \mu \mathrm{m}, \mathrm{g}-\mathrm{i}=13 \mu \mathrm{m}$. 

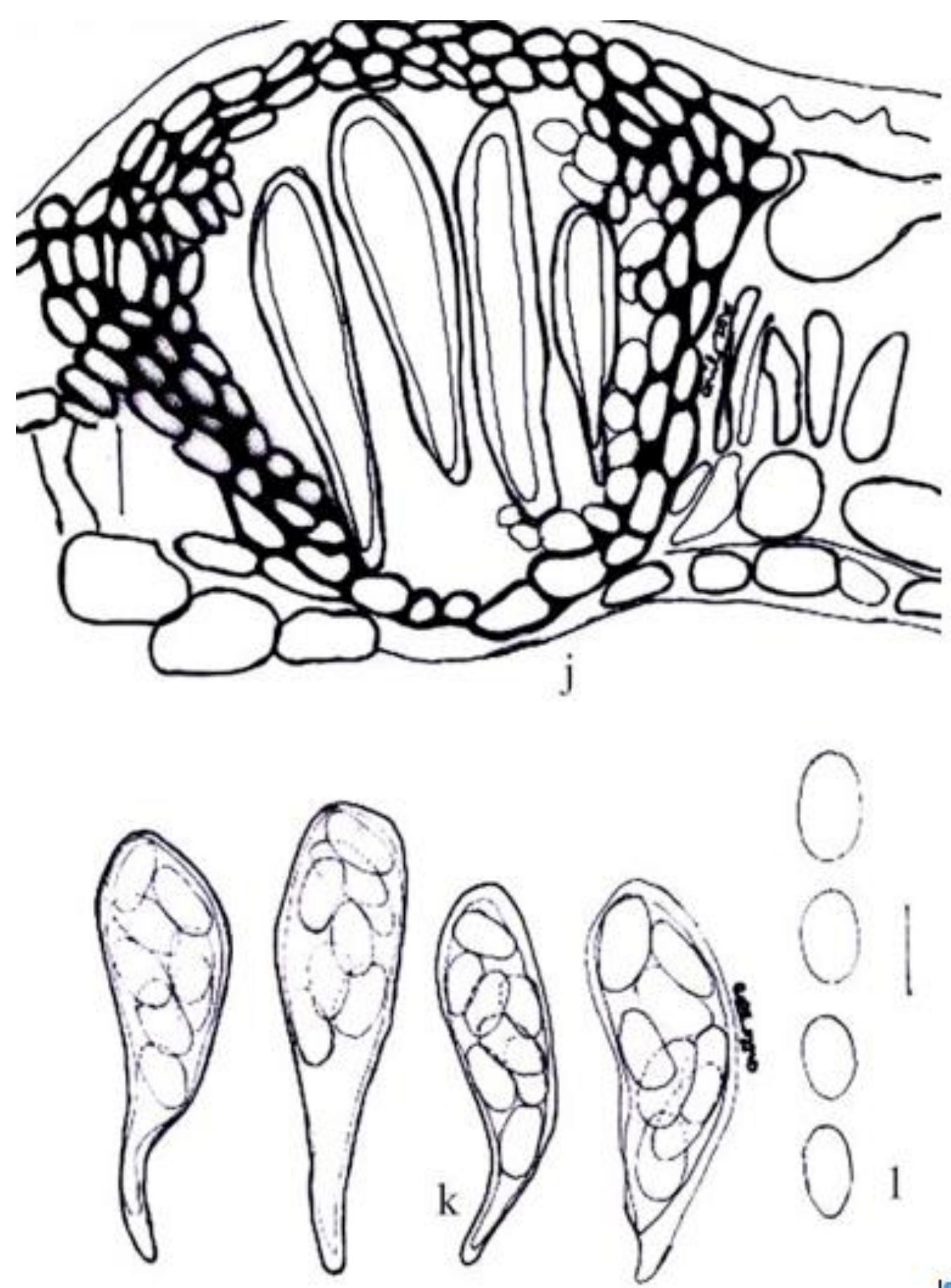

17

Figs 8j-m - Vestergrenia tetrazygiae (BPI 844972, from holotype of Guignardia tetrazygiae) line drawing. j, Section of ascoma in the leaf. k, Asci. 1, Ascospores. - Scale bars $=10 \mu \mathrm{m}$.

Hyponectria Sacc., Michelia 1(no. 2): 250 (1878)

Hyponectria cf. buxi (DC) Sacc. Michelia 1(2): 250 (1878)

(Fig. 10a-h)

= Guignardia rhamni Reusser Phytopathologische Zeitschrift 51: 235 (1959) MycoBank: MB 331548

= Guignardia cirsii Reusser Phytopathologische Zeitschrift 51: 235-236 (1964)

= Guignardia lonicerae Dearness \& Barthelow Mycologia 18: 245 (1926)

$=$ Laestadia steppani Petrak, Annales Mycologicy 18: 111 (1911)

= Guignardia steppani Petr. Annales Mycologici 32: 407 (1934)

Ascomata 70-140 $\mu \mathrm{m}$ diam., 50-100 $\mu \mathrm{m}$ high, on the surface of the leaf, perithecium, black, globose to sub-globose, immersed in plant tissues, coriaceous, solitary to clustered, ostiolate. Ostioles as black dots at the centre. Peridium 7-15 $\mu \mathrm{m}$ wide, one strata of 2 layers of cells textura 
angularis with thickened brown walls around ostiole. Paraphyses not observed. Asci 48-71 $\times 8-$ $13 \mu \mathrm{m}(\bar{x}=57 \times 9 \mu \mathrm{m}, \mathrm{n}=10), 8$-spored, unitunicate, cylindrical to cylindric-clavate, rounded at the apex, where the diam. is $8-10 \mu \mathrm{m}$, tapering gradually to a 4-19 $\times 4-6 \mu \mathrm{m}$ long pedicel attached to the basal peridium. Ascospores 11-14 $\times 3-5 \mu \mathrm{m}(\bar{x}=12 \times 4 \mu \mathrm{m}, \mathrm{n}=20)$, uniseriate or occasionally overlapping biseriate, falcate, fusiform, obovoid, ellipsoidal to oblong, hyaline, 1celled, coarse-guttulate, smooth-walled.

Material examined - SWITZERLAND, Von Vallon Betton, Massif de la Ste. Baume, var Frankreich on leaf of Rhamnus alpina, 3 June 1959, E. Müller, Botanische Sammlungen der Eidg. Technischen Hochschulezürich Flora des Aletschreservats 2965, Zürich (ETH 2965, from holotype of G. rhamni), SWITZERLAND, Unterster Teil gegen batch, Aletschwqld, Kanton Wallis on leaf of Cirsium spinossimum, 16 June 1963, E. Müller, Botanische Sammlungen der Eidg. Technischen Hochschulezürich Flora des Aletschreservats no number, Zürich (ETH, from holotype of G. cirsii),

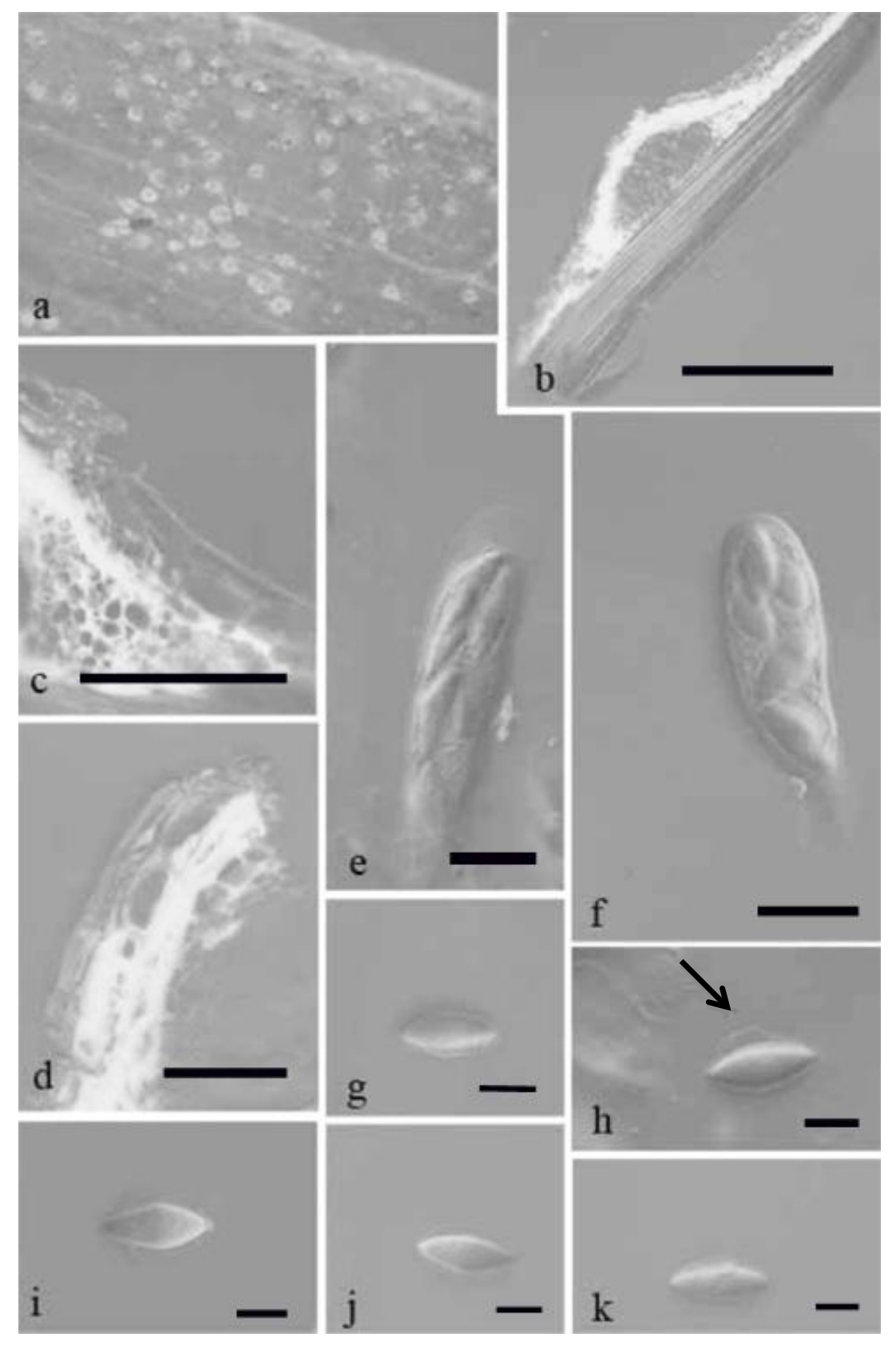

Figs 9a-k - Neodeightonia palmicola (KDH 1206, from holotype of Guignardia manokwaria) a, Ascomata on the host surface. b-d. Peridium comprising one strata of 2-3 layers of cells textura angularis with thickened brown walls. e, f. Asci. g-k. Ascospores (irregular wavy sheath, an arrowed). - Scale bars $\mathrm{b}=100 \mu \mathrm{m}, \mathrm{c}, \mathrm{d}=30 \mu \mathrm{m}, \mathrm{e}, \mathrm{f}=25 \mu \mathrm{m}, \mathrm{g}-\mathrm{k}=12 \mu \mathrm{m}$. 
USA, California, Alto Palo on leaf of Lonicera hispidula, 16 Mach 1918, E.T. Bartholow, New York Botanical Garden no number, New York (DAOM 5842, from holotype of G. lonicerae), CZECHOSLOVAKIA, Theusing, Bohemia, Bernkklau on leaf of Solidago virgaurea, June 1917, Steppan. R, Flora Bohemia and Moraviae Exsiccata No. 1232 (BPI 598475, from holotype of $G$. steppani).

Notes - With unitunicate asci and $\mathrm{J}^{+}$sub-apical ring, Guignardia cirsii and G. lonicerae are similar to Hyponectria Sacc. All species of Hyponectria are $\mathrm{J}+$ and $\mathrm{J}$-. Rarely, the diam. of ascomata is more than $200 \mu \mathrm{m}$ and ascospores more than $30 \mu \mathrm{m}$. There are only small differences between Hyponectria cf. buxi and the 4 species of Guignardia examined. The synopsis of the species is listed in table 1.
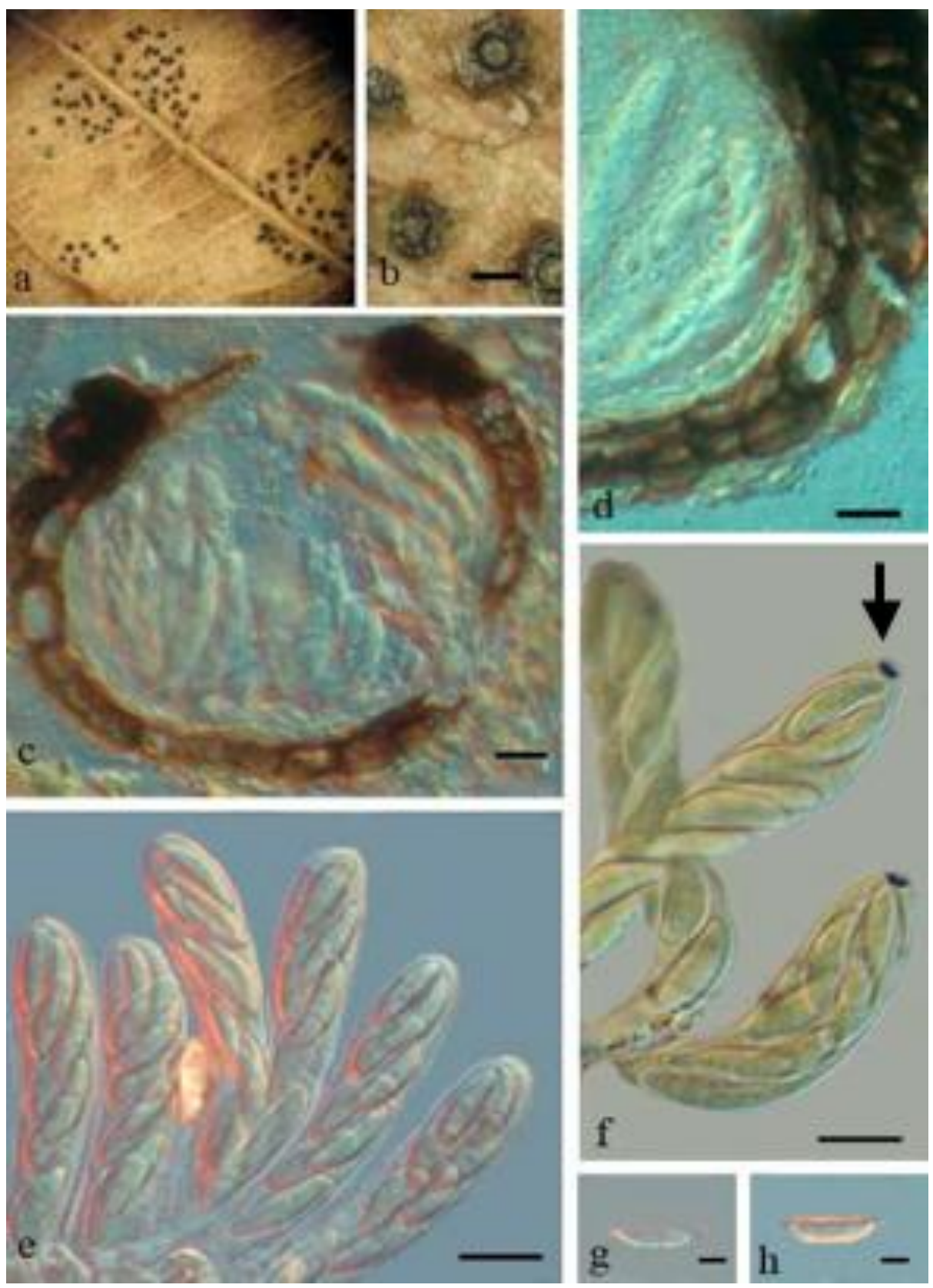

Figs 10a-h - Hyponectria cf. buxi (from holotype of G. rhamnii) a, Leaf spots on the leaves. b, Ascomata on the host surface. c, d. Peridium comprising one strata 2-3 layers of cells textura angularis with thickened brown walls and ostiole on the top. e, f. Asci, $\mathrm{J}^{+}$sub-apical ring by Meltzer (arrowed). g, h. Ascospores. - Scale bars $b=100 \mu \mathrm{m}, \mathrm{c}, \mathrm{d}=15 \mu \mathrm{m}, \mathrm{e}, \mathrm{f}=10 \mu \mathrm{m}, \mathrm{g}, \mathrm{h}=5$ $\mu \mathrm{m}$. 
Table 1 Synopsis of speices of Guignardia synonymised under Hyponectria cf buxi

\begin{tabular}{|c|c|c|c|}
\hline $\begin{array}{l}\text { Host plants and } \\
\text { Previous species nam }\end{array}$ & $\begin{array}{l}\text { Ascomata }(\mu \mathrm{m}) ; \\
\text { Peridium }(\mu \mathrm{m})\end{array}$ & $\begin{array}{c}\text { Asci }(\mu \mathrm{m}) ; \\
\text { AApical Ring } \\
\text { J- / J+ }\end{array}$ & $\begin{array}{l}\text { Ascospores } \\
(\mu \mathrm{m})\end{array}$ \\
\hline $\begin{array}{l}\text { Rhamnus alpina } \\
\text { (G. rhamni) }\end{array}$ & $70-140 \times 50-100 ; 7-15$ & $\begin{array}{l}48-71 \times 8-13 \\
\mathrm{~J}+(\mathrm{J} \text { plus })\end{array}$ & $11-14 \times 3-5$ \\
\hline $\begin{array}{l}\text { Cirsium spinosissimur } \\
\text { (G. cirsii) }\end{array}$ & $88-175 \times 88-175 ; 9-20$ & $\begin{array}{l}50-78 \times 8-15 \\
\mathrm{~J}+(\mathrm{J} \text { plus })\end{array}$ & $15-21 \times 5-6$ \\
\hline $\begin{array}{l}\text { Lonicera hispidula } \\
\text { (G. lonicerae) }\end{array}$ & $88-163 \times 100-163 ; 15-20$ & $\begin{array}{l}63-93 \times 13-17 \\
\mathrm{~J}-(\mathrm{J} \text { minus })\end{array}$ & $13-19 \times 5-8$ \\
\hline $\begin{array}{l}\text { Solidago virgaurea } \\
\text { (G. steppani) }\end{array}$ & $100-125 \times 95-125 ; 15-25$ & $\begin{array}{l}44-61 \times \\
10-17 ; \\
\text { J- (J minus })\end{array}$ & $21-20 \times 5-10$ \\
\hline
\end{tabular}

\section{Section 2: 290 Doubtful species (107 specimens found in poor condition and 183 unloaned} specimens, apparently missing and with invalid name)

Several holotype specimens of Phyllosticta obtained from various herbaria were found in poor condition, meaning lack of ascomata contents, absence of asci, ascospores and hamathecium. In some material, it was too less and inadequate to put into microscopic studies. Some of those specimens with ready-to-observe slides of type in fact contained no fungal material. Where cultures maintained as type, in some, the mats were in depauperate condition. In other cases, the repositories responded with message that specimens are lost, invalid or missing. Some of those national herbaria such as the Indian and China refused to loan the materials. All these taxa are treated here as doubtful species and listed below in Table 2.

Besides citations, wherever available, synonymy is provided to the species listed in Table 2. Present status of the species is elaborated based on microscopic observations and relevant literature.

Table 2 Doubtful species in "Guignardia" (sexual state of Phyllosticta)

\begin{tabular}{|c|c|c|c|}
\hline Name of fungus with authority, citation and synonymy & $\begin{array}{c}\text { Herbaria } \\
\text { /herbarium }\end{array}$ & Observation & Present status \\
\hline $\begin{array}{l}\text { 1. Guignardia abeana W. Yamam. \& K. Konno, Sci. rep. } \\
\text { Hyogo Univ. Agric. 5(1): } 9 \text { (1961) }\end{array}$ & $\begin{array}{l}\text { TNS, TFM, } \\
\text { NBRC, Japan }\end{array}$ & $\begin{array}{l}\text { Specimen } \\
\text { missing }\end{array}$ & $\begin{array}{l}\text { Illustration and } \\
\text { description by } \\
\text { original authors } \\
\text { (Yamam. \& } \\
\text { Konno, 1961) } \\
\text { indicate this as an } \\
\text { ascomycete; its } \\
\text { nature of asci not } \\
\text { discernible. }\end{array}$ \\
\hline $\begin{array}{l}\text { 2. G. abietella-sibirica (Schwarzman \& Tartenova) } \\
\text { Vasyag., in Vasyagina, Byzova \& Tartenova, Flora } \\
\text { Sporovykh Rasteni1 Kazakhstana [Cryptogamic Flora of } \\
\text { Kazakhstan], 12, Sumchatye. Griby Lokuloaskomitsety } \\
\text { (Loculoascomycetes) (Alma-Ata): } 25 \text { (1987) }\end{array}$ & LE, Russia & $\begin{array}{l}\text { Specimen } \\
\text { missing }\end{array}$ & $\begin{array}{l}\text { Curator of } \\
\text { herbarium } \\
\text { informed } \\
\text { specimen not } \\
\text { available (Pers. } \\
\text { comm.). }\end{array}$ \\
\hline 3. G. acaciae Hansf., Proc. Linn. Soc. N.S.W. 82: 14 (1957) & IMI, U.K & Examined & $\begin{array}{l}\text { Specimen in poor } \\
\text { condition; no } \\
\text { details available. }\end{array}$ \\
\hline 4. G. acerifera (Cook) Lindau, (1897) & BPI, U.S.A & Examined & $\begin{array}{l}\text { Specimen in poor } \\
\text { condition; no } \\
\text { details available. }\end{array}$ \\
\hline 5. G. adeana Rehm, Annls mycol. 10(6): 537 (1912) & F, U.S.A & Examined & $\begin{array}{l}\text { Sexual state of } \\
\text { Colletotrichum } \\
\text { species } \\
\text { (Glomerella } \\
\text { species); (Fig. } \\
11 \mathrm{a}-\mathrm{f} \text { ). }\end{array}$ \\
\hline
\end{tabular}


6. G. adianti (Höhn.) Arx \& E. Müll., Beitr. Kryptfl. IMI, U.K Schweiz 11(no. 1): 60 (1954)

7. G. adriatica Kirschst., Krypt.-Fl. Brandenburg (Leipzig) 7(3): 322 (1938)

B, Germany

8. G. aegyptiaca (Müll. Arg.) Reichert, Bot. Jb. 56: 669 (1921)

G, Switzerland

Not

examined

Examined (1916)

10. G. agerati Gonz. Frag. \& Cif. 1928., Estac. Agron. de MA, Spain Moca, B 13: 8 (1928)

11. G. ahlesiana (Hepp) Keissl., Rabenh. Krypt.-Fl., Edn 2 K, U.K (Leipzig) 8: 345 (1930)

12. G. ailanthi (Grove) Sacc., Syll. fung. (Abellini) 24(2): PAD, Italy 791 (1928)

13. G. alaskana M. Reed, Harriman Alaska Expedition: 34 (1902)

BPI, U.S.A

Examined

Not

examined

Not

examined

14. G. albicans Rehm, Leafl. of Philipp. Bot. 6: 2258 (1914)

15. G. alhagi Bubák, Annln K. K. naturh. Hofmus. Wien 28: 197 (1914)

16. G. alliacea Motohashi, Jun. Nishikawa \& C. Nakash., NBRC, Japan Mycoscience 49: 15-16 (2008)

17. G. alnea (Fr.) J. Schröt. (1894) (Ref. Index herbarium)

18. G. alnigena Y. Nisik. \& Watan., in Nogaku Kenkyu [Agriultural Research], Publ. Inst. Agric. Biol., Okayama Univ., 46: 208 (1959)

19. G. alternantherae (Sacc.) S. Hughes, Mycol. Pap. 48: 37 (1952)

20. G. alyxiae F. Stevens, Bulletin of the Bernice P. Bishop Museum, Honolulu, Hawaii 19: 101 (1925)

21. G. amomi S.M. Lin \& P.K. Chi, in Chi, [Fungal Diseases of Cultivated Medicinal Plants in Guangdong Province] (Guangdong): 43 (1994)
F, U.S.A

BPI, U.S.A

Not

examined

Examined
The specimen received from the herbarium was not the holotype.

Specimen not received from herbarium in spite of repeated requests. Curator informed the specimen has been loaned elsewhere.

The specimen receieved was not type material. Specimen in poor condition. Details not clear (Fig. 11 $\mathrm{g}-\mathrm{k})$.

Type specimen not available in $\mathrm{K}$. No

communication available from the herbarium.

Type seems to contain a lichen, Mastodia tessalata (Fig. 111-s)

Examined The specimen received was not holotype.

Examined The specimen received was not holotype.

No

communication available from the herbarium.

Specimen available; but without asci and ascospores.

Treated as uncertain species. No communbcation available from the herbaria.

NBRC \& YAM, examined Japan

PAD, Italy

Not

No

examined

BPI, U.S.A

Examined

HMAS, China

Not

examined

communbcation available from the herbarium.

Material received was not holotype. Repeated requests for holotype specimens remained unanswered.
Examined 
23. G. apiahyna (Speg.) Sacc., Syll. fung. (Abellini) 24(2): LPS, Argentina 779 (1928)

24. G. aphyllanthis Unamuno (1942) (Ref. Index fungorum)

25. G. apocyni (Ellis \& Everh.) M.E. Barr, Mycotaxon 46: NY, U.S.A $70(1993)=$ G. apocyni (Ellis \& Everh.) Vasyag.

26. G. araucariae Sousa da Câmara (1920)

LISE, Portugal

Not examined

27. G. arachidis Punith., Mycol. Pap. 136: 8 (1974)

IMI, U.K

Examined

28. G. araliae Gutner, Trudy Bot. Inst. Akad. Nauk SSSR, LE, Russia ser. 2, Sporov. Rast. 1: 289 (1933)

29. G. ardisiae Miura ex I. Hino \& Katum. (1967)

30. G. ardisiicola Motohashi, I. Araki \& C. Nakash.

31. G. arecae Sacc. (1917) (Ref. Index Fungorum)

32. G. arengae Rehm., Leafl. of. Philipp. Bot. 6: 2195 F, U.S.A (1914)

33. G. asparagi Gonz. Frag. \& Cif. (1926)

34. G. aspidistricola Motohashi, I. Araki \& C. Nakash., NBRC, Japan Mycoscience 49: 138-146 (2008)

35. G. astragali Nasyrov (1965) (Ref. Index Fungorum)

TNS, TFM,
Japan
NBRC, Japan
PAD, Italy

Not examined

Examined

Not

examined

Not

examined

Not examined species).

Specimen available; but without asci and ascospores.

Treated as uncertain species. No

communication received from herbaria.

Herbarium packet labeled as Leptosphaeria sp. Needs reexamination. No communication received from herbarium.

Dry culture specimen had no fungal diagnostic features. Treated as 'uncertain' species.

Holotype specimen contained immature fruiting bodies and hence treated as 'uncertain' taxon. No communication received from herbaria.

No communication received from herbarium. No communication received from herbarium. Seems to be Botryosphaeria sp. (Fig. 11t-z ${ }^{2}$ ). Needs recollection. The packaged received from MA without specimen. No communication received from herbarium. No communication received from 
36. G. bambusae I. Miyake \& Hara, Bot. Mag., Tokyo 24: TNS, 238 (1910)

Japan

37. G. bambusella Sacc., Notae Mycol. 23: 63 (1916)

38. G. bambusina Sacc., Atti Accad. Sci. Ven.-Trent.-Istr. PAD, Italy 10: 63 (1917)

39. G. bambusina Rehm, Leafl. of Philipp. Bot. 8: 2936 F, U.S.A (1916)

40. G. banonsensis (Petr.) Arx \& E. Müll., Beitr. Kryptfl. W, Austria Schweiz 11: 57 (1954)

41. G. betulae (Auersw.) Sacc. \& Trotter, Syll. fung. PAD, Italy (Abellini) 22: 77 (1913)

42. G. bidwellii (Ellis) Viala \& Ravaz, Bull. Soc. mycol. Fr. BPI, U.S.A 8: 63 (1892)

43. G. bidwellii f. bidwellii (Ellis) Viala \& Ravaz, Bull. BPI, U.S.A Soc. mycol. Fr. 8: 63 (1892)

44. G. bidwellii f. parthenocissi Luttr. (Ref. Index fungorum)

45. G. bidwellii var. bidwellii (Ellis) Viala \& Ravaz (1892)

BPI, U.S.A

46. G. biennis (Dearn.) Sacc., Syll. fung. (Abellini) 24(2): DAOM, Canada $780(1928)$

47. G. bispora Wulandari \& K.D. Hyde, Mycosphere 2(2): MFU, Thailand 115-128.

48. G. boltoniae Dearn. \& Barthol., Mycologia 18(5): 245 DAOM, Canada (1926)

49. G. bulgarica (Petr.) E. Mull., Revue Mycol., Paris 20: 4 MA, Spain (1955)

50. G. buxi (Fuckel) Lindau, Hilfsb. Sammelm Ascomyc. MA, Spain (Berlin): 21 (1938) herbarium Treated as non. inval.

(Invalid name).

No

communication received from herbaria.

No

Not
examined

communication received from herbarium.

Not No

examined

communication received from herbarium.

Examined Unknown ascomycete species (Fig. 12ag).

Examined Specimen received was not holotype.

Not No

examined communication received from herbarium.

Examined The specimen received was not holotype; herbarium packet labeled as Sphaerella bidwellii.

Not No

examined communication received from herbarium.

Not No

examined communication received from herbarium.

Not No

examined communication received from herbarium.

Examined Unidentified coelomycete species (Fig. 12hp).

Examined Only type available; needs re-collection for sequencing.

Examined Uncertain species, Botryosphaerialike (Fig. 12q-w).

Examined This is an unknown bitunicate ascomycete

Examined The specimen received was not holotype. 
51. G. buxicola Sousa da Câmara \& Luz, Agron. lusit. 1: 44 LISE, Portugal (1939)

52. G. cabelludae (Rangel) Sacc., Syll. fung. (Abellini) 24(2): 789 (1928)

53. G. cahirensis (J. Steiner) Sacc., Syll. fung. (Abellini) 24(2): 786 (1928)

54. G. calami (Syd.) Arx \& E. Müll., Beitr. Kryptfl. BPI, U.S.A Schweiz 11(no. 1): 55 (1954)

55. G. cambucae (Rangel) Sacc., Syll. fung. (Abellini) PAD, Italy 24(2): 789 (1928)

56. G. camelliae (Cooke) E.J. Butler ex Petch, Fungi of K, U.K India: 62 (1923)

57. G. canavaliae Cif. \& Gonz. Frag., Estac. Agron. de MA, Spain Moca, B 8: 19 (1927)

58. G. capsici Punith., Mycol. Pap. 136: 13 (1974)

59. G. caricae (Marchal \& Steyaert) Hendr., Publ. Inst. Natl. Agron. Congo Belge 35: 7 (1948)

60. G. caricae Sawada, Special Publication College of Agriculture, National Taiwan University 8: 57 (1959)

61. G. caricicola (Fuckel) Vasyag., in Vasyagina, Byzova \& Tartenova, Flora Sporovykh Rasteniı̌ Kazakhstana [Cryptogamic Flora of Kazakhstan], 12, Sumchatye. Griby Lokuloaskomitsety (Loculoascomycetes) (Alma-Ata): 26 (1987)

62. G. caricis Dearn. \& House. New York State Museum Bulletin 266: 73 (1925)

63. G. carpinea (Fr.) J. Schröt., in Cohn, Krypt.-Fl. NY, U.S.A Schlesien (Breslau) 3.2(3): 330 (1894) [1908]

64. G. caryophyllea (Cooke \& Harkn.) Wehm., Sydowia 6(5-6): 420 (1952)

65. G. cephalanthae Sawada, [as 'cepharanthae'], Special Publication College of Agriculture, National Taiwan University 8: 58 (1959)
Not

examined

RB (Brazil), Not

PAD (Italy) examined

Not

examined

Examined

Not

examined

Not

examined

Examined

IMI, U.K

Examined

BR, Belgium

Not

examined

NTU, Taiwan

Examined

G, Switzerland

Not

examined

DAOM, Canada

Examined

Not

examined

K, U.K

Not

examined

TNS, Japan
TFM, Not examined
No

communication received from herbarium.

No

communication received from herbaria.

No

communication received from herbaria.

The holotype contained limited asci and ascospores, which cannot clarify the identity.

No communication received from herbarium.

No

communication received from herbarium. Specimen was in poor condition and treated as uncertain species. Specimen was in poor condition and treated as uncertain species. No communication received from herbarium. Specimen was in poor condition and treated as uncertain species. No communication received from herbarium.

Seems to be Mycosphaerellalike taxon (Fig. $\left.12 \mathrm{x}-\mathrm{z}^{8}\right)$; needs recollection.

No communication or specimen received from herbarium No communication or specimen received from herbarium. No communication or specimen received 
66. G. cephalariae (Auersw.) F. Stevens, Trans. Ill. St. B, Germany Acad. Sci. 10: 184 (1917)

67. G. cephalariae var. alternantherae (Sacc.) F. Stevens, PAD, Italy Trans. Ill. St. Acad. Sci. 10: 184 (1917)

68. G. cephalariae var. cephalariae (Auersw.) F. Stevens, Trans. Ill. St. Acad. Sci. 10: 184 (1917)

69. G. cephalotaxi-nanae Sawada, Bull. Gov. Forest Exp. Stn 46: 117 (1950)

70. G. chandrapurensis P. G. Sathe \& K. M. Mogarkar, Marathwada University Journal of Science (Natural Science), B 16(9): 61 (1978) [1977]

71. G. chondri (H. L. Jones) Estee, Pubbl. Staz. Zool. UC, U.S.A Napoli 15: 378 (1936)

72. G. cinchonae Vincens, Bull. Soc. Path. vég. Fr. 9: 131 (1922)

73. G. circumscissa (Sacc.) Traverso, Fl. ital. crypt. 2: 381 PAD, Italy (1906)

74. G. clusiae F. Stevens, Trans. Ill. St. Acad. Sci. 10: 183 ILL, U.S.A (1917)

75. G. cocoicola Punith. Mycol. Pap. 136: 15 (1974)

76. G. coccocarpiae (Pat.) Sacc. \& Trotter, Syll. fung. PAD, Italy (Abellini) 22: 76 (1913)

77. G. codiae Thaung, Trans. Br. mycol. Soc. 66(1): 107 IMI, U.K (1976)

78. G. coffeae Punith. \& B.S. Lee, in Punithalingam, IMI, U.K Mycol. Pap. 149: 19 (1981)

79. G. coffeana (F. Noack) Sawada, J. Taihoku Soc. Agric. YAM, Japan 7(1): 23 (1943) [1942]

80. G. coronillae Sibilia, Annali Bot., Roma 18(2): 260 BPI, U.S.A (1929)

81. G. creberrima Syd. \& P. Syd., Philipp. J. Sci., C, Bot. DAR, Australia 8(5): 482 (1913)

82. G. convolvuli Gonz. Frag. \& Cif., Boln Real Soc. PAV, Italy
Examined

examined

Not

examined

from herbaria.

Specimen in poor condition.

No

communication or specimen received from herbarium.

No

communication or specimen received from herbarium.

No

communication or specimen received from herbaria.

Not

examined

No

communication or specimen received from herbarium.

Not No

examined communication or specimen received from herbarium.

Not No

examined communication or specimen received from herbarium.

Not No

exmained communication or specimen received from herbarium.

Examined Specimen contained an unknown ascomycete (Fig. 13a-g).

Not No

Examined communication or specimen received from herbarium.

Not No

examined communication or specimen received from herbarium.

Examined Specimen in poor condition. This species is a Phyllosticta sp. (Thaung, 1976).

Examined Specimen in poor condition. This is a Phyllosticta (Punith. \& Lee, 1981).

Not No

examined communication or specimen received from herbarium.

Examined Speciemen in poor condition.

Examined Specimen in poor condition. Not No 
Españ. Hist. Nat., Biologica 25: 359 (1925)

83. G. cookeana (Auersw.) Viala \& Ravaz ex Feltgen, B, Germany (1902)

84. G. cooperta (Desm.) Bubák, (1907)

85. G. corniculata (Hoffm.) Keiñl., Annln naturh. Mus. MW, Russia Wien 39: 195 (1925)

86. G. crepidis E. Müll., Sydowia 1: 213 (1957)

87. G. cryptomeriae Sawada, Bull. Gov. Forest Exp. Stn 45: $48(1950)$

88. G. cyperi P.B. Chavan \& Hosag., J. Econ. Taxon. Bot. 5(2): 447 (1984)

89. G. cytisi (Fuckel) von Arx \& E. Müller, KM, U.S.A Phytopathologische Zeitschrift 51: 232-233 (1964)

90. G. depressa (Peck) Dearn. \& House, (1925)

91. G. diapensiae (Rehm) Arx \& E. Müll., 11(1): 55 (1954)

92. G. dieffenbachiae Gonz. Frag \& Cif., Rep. Dominic. MA, Spain Est. Agron. Haina, Ser. B, Bot. Bull. 8: 21 (1927)

93. G. dieffenbachiae M.S. Ali \& Saikia, Indian Phytopath. 50(2): 201 (1997)

94. G. diffusa (Crié) Sacc. \& Trotter, Syll. fung. (Abellini) 22: 74 (1913)

95. G. dioscoreae Sawada [as '(Averna-Sacca)], Special Publication College of Agriculture, National Taiwan University 8: 58 (1959)

96. G. dioscoreae Sawada ex Katum., J. Jap. Bot. 40: 193 (1965) (Ref. Index fungorum; MycoBank) examined

communication or specimen received from herbarium.

No

exmained communication or specimen received from herbarium.

No

Not
examined

communication or specimen received from herbarium

Not No

examined communication or specimen received from herbarium No

examined communication or specimen received from herbarium. No NBRC and examined YAM, Japan

AMH, India

Not examined

Examined DAOM, Canada

Not examined

Examined

Examined

Not

examined

Not examined

YAM, Japan

Examined

TNS,

TFM, Not NBRC and examined YAM, Japan communication or specimen received from herbaria.

No

communication or specimen received from herbarium.

Contained Pleospora-like ascomycete (Fig. 13h-o).

No

communication or specimen received from herbarium. Specimen in poor condition.

Contained other ascomycete, possibly a secondary invader, Mycropeltis-like (Fig. 13p-u).

No communication or specimen received from herbarium. No communication or specimen received from herbarium.

Specimen contained a Colletotrichum $\mathrm{z}^{2}$ ). Treated as invalid name (Index Fungorum). No communication or specimen received species (Fig. 13v- 
97. G. dioscoreae A.K. Pande, Sydowia 22(5-6): 367 AMH, India (1969) [1968]

98. G. dioscoreae-bulbiferae A. Pande, Ascomycetes of Peninsular India (Jodhpur): 36 (2008) (Ref. Index Fungorum and MycoBank)

99. G. dodartiae Nasyrov ex Vasyag., in Vasyagina, Byzova \& Tartenova, Flora Sporovykh Rasteniı̌ Kazakhstana [Cryptogamic Flora of Kazakhstan], 12, Sumchatye. Griby Lokuloaskomitsety (Loculoascomycetes) (Alma-Ata): 29 (1987)

100. G. dracaenae Gutner, Acta Inst. bot. Komarov. Acad. LE, Russia

Sci., Pl. Crypt, ser. 2 11(1): 296 (1933)

101. G. durmitorensis Bubák, Bot. Közl. 14(3-4): 55 (1915)

102. G. dyerae Punith. \& P. H. Wong, in Punithalingam, Mycol. Pap. 149: 28 (1981)

103. G. echinophila (Schwein.) Traverso, Fl. ital. crypt. 2: 390 (1907)

104. G. ellipsoidea Wulandari \& K.D. Hyde, Mycosphere 2(2): 119 (2011)

105. G. empetri (Rostr.) Lar.N. Vassiljeva, Pirenomits. Lokuloaskomits. Severa Dal'nego Vostoka (Leningrad): 78 (1987)

106. G. epilobii (Wallr.) Lindau, Mycologia 38(2): 152 NY, U.S.A (1946) (1946)

107. G. ericetorum (Körb.) Rambold \& Triebel = Rhymbocarpus ericetorum (Körb.) Etayo, Diederich \& Ertz, in Diederich, Ertz \& Etayo, Lichenologist 42(3): 266 (2010)

108. G. eucrypta Petr., Beitr. Kryptogamenfl. Schweiz, W, Austria 11(172) (1954)

109. G. eugeniae S.M. Lin \& P.K. Chi, in Chi, [Fungal Diseases of Cultivated Medicinal Plants in Guangdong Province] (Guangdong): 62 (1994)

110. G. eupatorii Punith., Mycol. Pap. 136: 21 (1974)

111. G. euphorbiae Rayss, Bull. rimmest. Soc. Mycol. Fr. 62: 20 (1946)

112. G euphorbiae T. M. Achundov, : 5 (1971)
Not

Examined

Not

Examined

LE, Russia

Not

examined

IMI, U.K

PH, NY, U.S.A

MFU, Thailand

C, Denmark

$\mathrm{L}$, Netherlands The

Not

examined

Examined

HMAS, China

Not

examined

IMI, U.K

Examined

Not

(Romania), PC examined (France)

BPI, U.S.A

examined from herbaria.

Treated as nom. inval. (Invalid name) (Index fungorum).

\section{No} communication or specimen received from herbarium.

No

communication or specimen received from herbarium. Treated as nom. inval. (Invalid name).

No communication or specimen received from herbarium.

Examined Specimen in poor condition.

Not No specimen received from herbarium. Specimen in poor condition.

No

communication or specimen received from herbaria.

No type culture, need recollecting and sequencing. No communication or specimen received from herbarium.

No communication or specimen received from herbarium. No communication or specimen received from herbarium. Specimen in poor condition.

No

communication or specimen received from herbarium. The ex-type was without asci and ascospores.

No

communication or specimen received from herbaria.

No

Not No

examined communication or 
113. G. euphorbiae-spinosae Bubák, Bot. Közl. 14(3-4): 56 (1915)

114. G. excentrica (Crié) Sacc. (Ref. Index Fungorum)

115. G. excentrica var. excentrica (Crié) Sac.

116. G. excentrica var. major Bat., Publções Inst. Micol. URM, Brazil Recife 284: 9 (1960)

117. G. fagi H. J. Huds., Nova Hedwigia 10(3/4): 323 IMI, U.K (1966) [1965]

118. G. fatsiae I. Hino \& Katum., Apud Katumoto, J. Jap. YAM, Japan Bot. 40: 194 (1965)

119. G. fici W. Y. Li \& W. Y. Zhuang, Mycotaxon 100: HMAS, China $213(2007)$

120. G. fici-beecheyanae Sawada, J. Toihaku. Soc. Agric. YAM, Japan 7: 123 (1942)

121. G. fici-septicae Sawada in J. Taihoku Soc. Agric., NTU, Taiwan 7:126 (1942)

122. G. fici-septicae Sawada ex W.Y. Li \& W.Y. Zhuang, HMAS, China in Li \& Zhuang, Mycotaxon 100: 214 (2007)

123. G. fimbriata sensu auct. brit.,

124. G. fimbriatae (Vain.) Keissl., Rabenh. Krypt.-Fl. TUR, Finland (Leipzig) 8: 346 (1930).

125. G. flacourtiae Anahosur, Sydowia 24(1-6): 169 (1971)

IMI, U.K

126. G. franconica (Petr.) E. Müll., Sydowia 1: 213 (1957)

W, Austria

127. G. freycinetia Rehm, Philipp. J. Sci., C, Bot. 8(2): 184 (1913) specimen received from herbarium. Specimen in poor condition.

No

Not

examined

communication or specimen received from herbarium.

Not No

examined

Not

examined

Not

examined

Examined

Not

examined

Not

examined

Examined

Not

examined

Not

examined

Examined

Examined

Examined

Examined communication or specimen received from herbarium.

No

communication or specimen received from herbarium.

No

communication or specimen received from herbarium.

Found to be a

Colletotricum species (Fig. 14aw).

No

communication or specimen received from herbarium.

No

communication or specimen received from herbarium.

Specimen was without asci and ascospores.

Treated as uncertain species. No

communication or specimen received from herbarium. Treated as nom. inval. (Invalid name. Art 36.1). (Index fungorum) Sensu auct brit.

Specimen was without asci and ascospores; treated as uncertain species. Specimen in poor condition. This is a Phyllosticta. Specimen contained an ascomycete; but not Phyllosticta (Fig. 14f-m). Specimen in poor condition; treated as uncertain 
128. G. fulvida F.R. Sand., N.Z. J1 agric. Res. 8(1): $139 \begin{aligned} & \text { PDD, } \\ & \text { Zealand }\end{aligned}$ New Examined (1965)

129. G. fuscocinerea Rehm, Leafl. of Philipp. Bot. 6: 2195

(1914)

130. G. fuscocoriae Rehm, Leafl. of Philipp. Bot. 6: 2195 ILL, U.S.A

(1914)

131. G. galactina Dearn. \& Barth. New York State Museum Bulletin 179 (1915)

132. G. gentianicola (DC.) Arx \& E. Müll., 11(1): 50 (1954)

133. G. gloiopeltidis Miyabe \& Tokida, Bot. Mag., Tokyo 61: 118 (1948)

134. G. glycyrrhizae Antok., sec Petr. 1934, Annal. Mycol. 32: 339 (1934)

135. G. gmelinae Ts. Kobay., Trans. Mycol. Soc. Japan 21(3): 314 (1980)

136. G. graminea Lobik, Materialy po floristicheskim i faunisticheskim obsledovaniyam Terskogo okruga [Data from investigations on the flora and fauna of the Ter region], 255 (1928)

137. G. graminis (Lind) M.E. Barr, 73: 12 (1959)

138. G. harunganae Sivan. \& Okpala, Trans. Br. mycol. IMI, U.K Soc. 72(3): 522 (1979)

139. G. heliconiae Gonz. Frag. et Cif., Boletin Real Soc. MA, Spain Espan. Hist. Nat. Madrid 27(325) 1928

140. G. herbarum Vasyag., in Vasyagina, Byzova \& Tartenova, Flora Sporovykh Rasteniı̌ Kazakhstana [Cryptogamic Flora of Kazakhstan], 12, Sumchatye. Griby Lokuloaskomitsety (Loculoascomycetes) (Alma-Ata): 30 (1987)

TNS, TFM, Not

(Kazakhztan), species.

Specimen without asci and ascospores.

Treated as uncertain species. No

examined communication or specimen received from herbaria.

Examined Specimen in poor condition; treated as an unknown coelomycete (Fig. 14n-q).

DAOM, Canada Examined This specimen is seems to be Hyponectria (Wang \& K.D. Hyde 1999).

No

Not
examined

TNS, TFM, Not NBRC and examined YAM, Japan NBRC and examined YAM, Japan

TNS, TFM, Not NBRC and examined YAM, Japan

TNS, TFM, Not NBRC and examined YAM, Japan

B, Germany

Not examined

AA LISE (Portugal), LE (Russia)
Examined

Examined

communication or specimen received from herbarium. Specimen referred as Didymella gloiopeltidis (Miyabe \& Tokida) Kohlm. \& E. Kohlm. (1979).

No

communication or specimen received from herbaria.

Repeated requests for holotype specimen to several herbaria, remained unanswered.

Repeated requests for holotype specimen to several herbaria remained unanswered.

No

communication or specimen received from herbarium. Specimen in poor condition. This is now a Phyllosticta (Sivanesan \& Okpala 1979). Specimen in poor condition.

Not No

examined communication or specimen received from herbaria. 
141. G. hernandiae Tak. Kobay. \& Kawabe, Japanese Journal of Tropical Agriculture 36(3): 201 (1992)

142. G. heterostemmae (T. S. Ramakr. \& K. Ramakr.) D. AMH, India R. Pawar \& J. N. Kapoor (1989)

143. G. heterostemmatis (T. S. Ramakr. \& K. Ramakr.) D. AMH, India R. Pawar \& J. N. Kapoor (1989)

144. G. heterotrichi Stevens, Transaction of the Illinois Academy of Science 10: 182-183 (1917)

145. G. heveae Gonz. Frag. \& Cif., Boln Real Soc. Españ. Hist. Nat., Biologica 26: 492 (1926)

146. G. heveae Syd. \& P. Syd., Annls mycol. 14(5): 360 (1916)

147. G. hibisci-sabdariffae Sawada, Special Publication College of Agriculture, National Taiwan University 8: 59 (1959)

148. G. himalayensis E. Müll., Phytopath. Z. 34(4): 414 (1959)

149. G. hispanica Bubák \& Gonz. Frag., Hedwigia 57: 4 BPI, U.S.A (1915)

150. G. horaninovia Nasyrov [as 'horaninowiae'], Griby Oazisov Vostochnoĭ Turkmenii (Ashkhabad): 300 (1965)

151. G. humulina Bubák, Annls mycol. 4(2): 110 (1906)

152. G. ilicis (Jacz.) Schrantz, Bull. trimest. Soc. mycol. Fr. P, France 76(4): 327 (1961)

153. G. ilicis-formosanae Sawada, Rep. Govt Res. Inst. Dep. Agric., Formosa 85: 30 (1943)

154. G. ingae F. Stevens, Annls mycol. 28(5/6): 367 (1930)
LPS (Argentina), Not PAV (Italy) examined

DAR, Australia

NTU, Taiwan

ZT, Zwitzerland

Examined

Not

examined

Not

examined

BPI, U.S.A

Not

exmained

Not

exmained

TNS,

TFM, Not NBRC and examined YAM, Japan

ILL, U.S.A

Examined
Repeated requests

for holotype

specimen to

several herbaria

remained

unanswered.

Repeated requests

for holotype specimen to

herbarium,

remained

unanswered.

Repeated requests

for holotype specimen to herbarium, remained unanswered. This specimen seems to be a Hyphonectria (Sivanesan \& Shivas, 2002; Wang \& Hyde, 1999).

No

communication or specimen received from herbaria.

The species belong to Phomopsis (Fig. 14r-w).

No

communication or specimen received from herbarium.

This is a Kabatia species (Fig. 15ag) (Müller, 1959).

No

communication or specimen received from herbaria.

No

communication or specimen received from herbarium. No communication or specimen received from herbarium.

No

communication or specimen received from herbarium. No communication or specimen received from herbaria.

The specimen was labeled as Irenopsis ingae 
155. G. insularis sensu auct. brit.

156. G. insularis (A. Massal.) Keissl., Rabenh. Krypt.-Fl., VER, Italy Edn 2 (Leipzig) 8: 343 (1930)

157. G. irritans Setch. \& Estee, University of Calif. Publ. BPI, U.S.A Bot. 4: 311 (1913)

158. G. istriaca Bubák, Annls mycol. 14(1/2): 12 (1916)

BPI, U.S.A

159. G. istriaca Kirschst., Annls mycol. 34(3): 187 (1936)

160. G. jasmini (Petch) Sacc., Syll. fung. (Abellini) 24(2): 789 (1928)

161. G. jasminicola Gonz. Frag., Intr. Flor. Microm. Catal, MA, Spain 83 (1917)

162. G. javanica Koord., Atti Ist. bot. R. Univ. Pavia, 2 Sér. BO, Indonesia 13: 206 (1907)

163. G. juniperina (Ellis) Rota-Rossi, Atti Ist. bot. R. Univ. NY, U.S.A Pavia, 2 Sér. 13: 206 (1907)

164. G. jussiaeae F. Stevens, Bulletin of the Bernice P. ILL, U.S.A Bishop Museum, Honolulu, Hawaii 19: 101 (1925)

165. G. kareliniae Nasyrov, Griby Oazisov Vostochnoı̌ LE, Russia Turkmenii (Ashkhabad): 300 (1965)

166. G. laricina Sawada, Report of the Department of NTU, Taiwan Government Research Institute of Formosa 86: 10 (1943)

167. G. latemarensis E. Müller Phytopathologische Zeitschr. ETH,

Switzerland

168. G. linderae Sawada, Report of the Department of NTU, Taiwan Government Research Institute of Formosa 86: 10 (1943)

169. G. linderae Sawada ex. W. Y. Li \& W. Y. Zhuang in I HMAS, China Mycotaxon 100: 216 (2007)

170. G. lingue (Speg.) Sacc. \& Trotter, Syll. fung. (Abellini) PAV, PAD, Italy 22: $74(1913)$

(F. L. Stevens \& Tenon) F. L.

Stevens (Fig. 15ho).

Not

examined

Not

examined

Not

examined

Not

examined

Not

examined

Not

examined

Not

examined

Not

examined

Not

examined

Examined

Not

examined

Examined

Examined

Examined

Not

examined

Not

examined
Sensu auct. brit.

No

communication or specimen received from herbarium.

No

communication or specimen received from herbarium.

No

communication or specimen received from herbarium.

No

communication or specimen received from herbaria.

Currently as nom. illegit. 53.1 (Index Fungorum).

No

communication or specimen received from herbaria.

No

communication or specimen received from herbarium. Specimen not available (pers. comm. A. Rifai). No communication or specimen received from herbarium.

This is an unknown unitunicate ascomycete (Fig. 15p-w).

No

communication or specimen received from herbarium. Specimen in poor condition.

This is seems to be a Hyponectria species (Wang \& Hyde 1999).

Specimen in poor condition.

No

communication or specimen received from herbarium.

No communication or 
171. G. lini (Rostr.) Sacc \& Trotter, Syll. fung. (Abellini) 22: 72 (1913)

172. G. lonchocarpi (Marchal \& Steyaert) Hendr., Publ. Inst. BR, Belgium Congo Belge 35: 7 (1948)

173. G. lysimachiae Jaap, Fung. Saxon. Exsicc., Pilze Sachsen's: no. 2262 (1914)

174. G. lunulata (Rostr.) Larsen in Rosenvinge \& Warming, C, Denmark in Botany of Iceland 2(3): 832, 9 (Fungi of Island) (1932)

175. G. maculiformis (Pers.) Mig., (1913)

NY, U.S.A

176. G. magnolia (Schwein.) J.H. Mill (1941), Mycologia 33 PH, U.S.A (1941)

177. G. mammeae Cif. \& Gonz. Frag., Boln de la Real Soc.

Españ. Hist. Nat., Madrid 26: 193, fig. 1 (1926)

178. G. mangiferae A. J. Roy, Indian Phytopath. 20(4): 348 AMH, India (1968) [1967]

179. G. maninotis var. cajani Sacc., Atti Soc. Veneto-Trent. BPI, U.S.A

Sci. Nat., Padova 23: 63 (1917)

180. G. manihotis var. deminuta Sacc., Nuovo G. bot. ital. PAD, Italy

23(2): 200 (1916)

College of Agriculture, National Taiwan University 8: 59 (19
181. G. manihotis var. manihotis Sacc. [as 'manihoti'], Annls mycol. 12(3): 304 (1914) (1914)

182. G. manihoticola Sawada ex W.Y. Li \& W.Y. Zhuang, HMAS, China in Li \& Zhuang, Mycotaxon 100: 216 (2007)

183. G. manihoticola Sawada, Special Publication

TNS, Japan

$$
\text { PAD, Italy }
$$

184. G. medinillae (Rangel) Sacc., Syll. fung. (Abellini PAD (Italy), RB (Brazil)

PC, France

185. G. melanostigma (Lév.) Sacc. \& Trotter, Syll. fung. (Abellini) 22: 75 (1913)

186. G. mespilii (Fautrey) Schrantz, in Bull. Soc. Mycol. Fr. NY, U.S.A 76 (4): 327 (1961)

187. G. miconiae Seixas \& R. W. Barreto, in Seixas, Barreto \& Killgore, Mycologia 99(1): 103 (2007)

188. G. microsticta Sacc., Syll. fung. (Abellini) 22: 75 (1913), PAD, Italy
Not

examined

Examined

Not

specimen received from herbaria. Specimen in poor condition.

Not No

examined communication or specimen received from herbarium.

No

communication or specimen received from herbarium.

Examined No

communication or specimen received from herbarium.

Not

examined

communication or specimen received from herbarium.

Examined The specimen was in poor condition.

Examined Specimen in poor condition.

Examined Specimen in poor condition.

Examined Specimen in poor condition

Not

examined

communication or specimen received from herbarium

Not No

examined communication or specimen received from herbarium.

No

communication or specimen received from herbarium.

The specimen contained sexual state of Colletotrichum sp. (Fig. 15x-z ${ }^{8}$.

No

communication or specimen received from herbaria.

No

Not

examined

communication or specimen received from herbarium.

Not No

examined communication or specimen received from herbarium.

Examined Specimen in poor condition.

Not No

examined communication or specimen received 
189. G. microthelia (Wallr.) Keissl., Rabenh. Krypt.-Fl.,

Edn 2 (Leipzig) 8: 344 (1930)

190. G. migrans (Rehm) K.D. Hyde, Sydowia 47(2): 192 (19 F, U.S.A

191. G. mildae Treigienè, Mikol. Fitopatol. 40(5): 428 (2006)

192. G. millepunctata (Desm.) Lind., Danish Fungi

(Copenhagen): 201 (1913)

193. G. minuta Arx \& Müll., Beitr. Kryptfl. Schweiz 11 (no. 1): 58 (1954)

194. G. mirabilis E. Müll., Phytopath. Z. 34(4): 412 (1959)

195. G. miribelii Aa, Persoonia 8(3): 283 (1975)

196. G. moelleriana Sousa da Câmara [as 'molleriana'], Bolm Soc. broteriana, Coimbra, sér. 1 25: 6 (1910)

197. G. musae Syd. \& P. Syd., Annls mycol. 10(1): 80 (1912 F, U.S.A

198. G. musicola Wulandari, L. Cai and K.D. Hyde, in

Wulandari, To-anun, Cai, Abd-Elsalam \& Hyde,

Cryptog. Mycol. 31(4): 412 (2010)

199. G. myopori Sousa da Câmara, Agron. lusit. 10(4): 287 LISE, Portugal (1948)

200. G. nectandrae F. Stevens, Bot. Gaz. 69: 255 (1920)

201. G. niesslii (Kunze ex Rehm) Lindau, Syll. fung. (Abellir BPI, U.S.A

1: 421 (1881)

202. G. nilagirica T. S. Ramakr. \& K. Ramakr.,

Proc. Indian natn Sci. Acad., Part B. Biol. Sci. 28: 56 (1948)

203. G. oleandrina (Curzi \& Barbaini) Arx \& E. Müll., in Beitr. Kryptogamenfl. Schweiz 11: 174 (1954)

204. G. olivieri (Vouaux) Sacc., Syll. fung. (Abellini) 24(2): ‘ NY (U.S.A) (1928)
ILL, U.S.A

Not

examined

Examined

MW, Russia

$\mathrm{BR}$, Belgium

Not

examined

Examined

CBS, Netherlands

Not examined

Not

examined

Examined

Examined

MFU, Thailand

Not

examined

Examined

Not

examined

AMH, India

Not

examined

ZT Switzerland

Not

examined

Not

examined from herbarium. No

communication or specimen received from herbarium. Specimen in poor condition.

No

communication or specimen received from herbarium.

No

communication or specimen received from herbarium.

This is an unknown ascomycete.

This is a Kabatia species (Fig. 16ag) (Müller 1959). No

communication or specimen received from herbarium. According to Aa \& Vanev (2002) this is not Phyllosticta.

No

communication or specimen received from herbarium The specimen is in poor condition.

No ex-type

culture, needs recollecting and sequencing.

No

communication or specimen received from herbarium

Dothidea-like species (Fig. 16hp)

No

communication or specimen received from herbarium. No

communication or specimen received from herbarium. No

communication or specimen received from herbarium. No

communication or specimen received from herbarium. 
205. G. opuntiae Petch, Ann. R. bot. Gdns Peradeniya 9: 317 K, NY (U.S.A) (1925)

206. G. oosperma Kirschst., Krypt.-Fl. Brandenburg (Leipziє̨ B, Germany 7(3): 320 (1938)

207. G. oxyriae (Rostr.) P. Larsen. The Botany of Iceland II p C, Denmark 9: $484(1932)$

208. G. paulowniae (S. Ito \& Kobayasi) W. Yamam. \& S. Ito TNS Sci. Rep. Hyogo Univ. Agric. 5(1): 11 (1961)

209. G. pedrosensis Bubák \& Gonz. Frag., Hedwigia 57: 4 (1915)

210. G. pegani (Rostr.) Sacc. \& Trotter, Syll. fung. (Abellini) C, Denmark 22: $72(1913)$

211. G. perpusilla (Desm.) Verpl., Bull. Jard. bot. État Brux. NY (U.S.A) and 5: 340 (1939)

212. G. perseae Punith., Mycol. Pap. 136: 43 (1974)

213. G. photiniae J. V. Almeida \& Sousa da Câmara,

Bolm Soc. broteriana, Coimbra, sér. 1 24: 11 (1909)

214. G. phytolaccae J. V. Almeida \& Sousa da Câmara,

Bolm Soc. broteriana, Coimbra, sér. 1 24: 11 (1909)

215. G. pinastri (DC.) Lindau, in Engler \& Prantl, Nat.

Pflanzenfam., Teil. I (Leipzig) 1(1): 423 (1897)

216. G. pini Sivan., Trans. Br. mycol. Soc. 73(1): 169 (1979) IMI, U.K

217. G. piperis (Rehm) Arx \& E. Müll., Beitr. Kryptfl.

Schweiz 11(no. 1): 56 (1954)

218. G. plectroniae Syd. and P. Syd., Annls mycol. 15(3/4):

207 (1917)

219. G. pleurothallis Dearn. \& House, Bull. N.Y. St. Mus. 266: 72 (1925)

220. G. podocarpi Crous in Seifert \& Castañeda Ruíz, S. Afr. J. Bot. 62(2): 90 (1996)

221. G. polygonati (Schwein.) Lindau, in Engler \& Prantl, Nat. Pflanzenfam., Teil. I (Leipzig) 1(1): 423 (1897)

222. G. polygoni Reusser, Phytopath. Z. 51: 236 (1964)
NBRC

TFM, Not

YAM, Japan

C, Denmark

Not

examined

Examined

Examined BR (Belgium)

Not

examined

IMI, U.K

Not

examined

LISE, Portugal

Not

examined

LISE, Portugal

Not

examined

NY, U.S.A

Not

examined

Examined

F, U.S.A

F. U.S.A

DAOM, Canada

Not

examined

PREM, South Examined Africa

NY, U.S.A

Not

examined

ETH, Ethiopia

Not
No

communication or specimen received from herbaria.

Specimen in poor condition.

Speciemn contained an unknown ascomycete (Fig. 16q-v).

No

communication or specimen received from herbaria.

No

communication or specimen received from herbarium.

Botryosphaerialike species (Fig. $16 \mathrm{w}-\mathrm{z}^{2}$ )

No

communication or specimen received from herbaria.

No

communication or specimen received from herbarium.

No

communication or specimen received from herbarium.

No

communication or specimen received from herbarium.

No

communication or specimen received from herbarium.

Speciment in poor condition.

Specimen in poor condition.

This is a sexual state of Colletotrichum sp. (Fig. 17a-h).

No

communication or specimen received from herbarium. Specimen in poor condition.

No communication or specimen received from herbarium. No

examined communication or specimen received 
223. G. polygoni-chinensis Sawada ex W.Y. Li \& W.Y.

Zhuang, in Li \& Zhuang, Mycotaxon 100: 218 (2007)

224. G. polygoni-chinensis Sawada, in J. Taihoku Soc. Agric NTU, Taiwan

$7: 124(1942)$

225. G. populi G. E. Thompson. Mycologia 46: 658 (1954)

226. G. poterii (Petr.) E. Müll., in Sydowia, Beih.

1(Petrak-Festscrift): 213 (1957)

227. G. prasiolae (G. Winter) Lemmerm., Naturwiss.

Ver. Bremen 17: 199 (1901)

228. G. prominens Earle, Muhlenbergia 1: 15 (1901)

229. G. pruni-persicae Sawada, Report of the Department of Agriculture, Government Research Institute of Formosa 85 230. G. psidii Ullasa \& Rawal, Curr. Sci. 53(8): 436 (1984)

231. G. psoromoides (Borrer) Keissl., Rabenh. Krypt.-Fl., Edn 2 (Leipzig) 8: 346 (1930)

232. G. puerariae Sawada, Report of the Department of Agriculture, Government Research Institute of Formosa 86: 11 (1943)

233. G. puiggarii (Speg.) Sacc. [as 'puiggari'], Syll. fung. (Abellini) 24(2): 788 (1928)

234. G. pullulans Kleb., Ber. dt. bot. Ges. 42: 69 (1924)

235. G. punctiformis Chardon, Mycologia 32: 178 (1940)

236. G. punctoidea (Cooke) J. Schröt, Pilzfl. Schlesiens

2: 329 (1894)

237. G. pyricola (Nose) Yamam. [as 'piricola'], Sci. Rep. Hyogo Univ. Agric., Ser. Agr. Biol. 5(1): 11 (1961)

238. G quercus-ilicis Traverso, Fl. ital. crypt. 2: 390 (1907)

239. G. ramulicola (Pass.) M. Morelet, Ann. Soc. Sci. Nat. Arch. Toulon et du Var 21: 105 (1969)
BPI, U.S.A

ZT, Zwitzerland

Not

examined

from herbarium.

No

communication or specimen received from herbarium.

Examined Specimen in poor condition.

Examined This is a

Physalospora sp.

(Fig. 17i-s).

No

examined communication or specimen received from herbarium.

B, Germany

Not

examined

No

communication or specimen received from herbarium.

BPI, U.S.A Examined

Specimen in poor condition.

NTU, Taiwan Examined

CBS, The

Netherlands examined

K, U.K

Not

examined

TNS, TFM, Not

NBRC and examined

YAM, Japan

LPS, Argentina

Not

examined

B, Germany

Not

examined

VIC, Brazil Examined

K, U.K Examined

TNS, TFM, Not

NBRC and examined

YAM, Japan

PAV, PAD, Italy

Not

examined

PARMA, Italy

Not

examined
Specimen in poor condition.

No

communication or specimen received from herbaria.

This is

Phyllosticta

(Glienke et al.

2011)

No

communication or specimen received from herbarium.

No

communication or specimen received from herbaria

No

communication or specimen received from herbarium.

No

communication or specimen received from herbarium. Specimen in poor condition.

Specimen in poor condition.

No

communication or specimen received from herbaria.

No

communication or specimen received from herbaria.

No

communication or specimen received from herbarium. capitalensis 
240. G. rathenowiana Kirschst., Krypt.-Fl. Brandenburg

(Leipzig) 7(3): 322 (1938)

241. G. reniformis Prill. \& Delacr., C. r. hebd. Séanc. Acad. B, Germany Sci., Paris (1900)

242. G. rhodomyrti Sawada, in J. Taihoku Soc. Agric., 7:124 NTU, Taiwan (1942)

243. G. rhodorae (Cooke) B.H. Davis, Mycologia 38(1): 48 (1946)

244. G. rhytismoides (Bab.) Zahlbr., 7(nos 601-700): 618

(1931)

BPI, U.S.A

Not

examined
245. G. robiniae S. Ito \& Kobayasi, Bull. Govt Forest Exp. Stn Meguro 108: 18 (1958)

246. G. rosae (Auersw.) Petr., Annls mycol. 19(1/2): 110 (1921)

247. G. rosaecola Feltgen, (1903)

(Ref. Index Fungorum)

248. G. rosicola Feltgen (1903) (Ref. Index Fungorum)

249. G. rhytismophila Rehm, Annls mycol. 4(1): 70 (1906)

F, U.S.A

250. G. rhynchosporae F. Stevens, Trans. Ill. St. Acad. Sci. 10: 162-218 (1917)

251. G. ryukyuensis I. Hino \& Katum, In Bull. Fac. Agric. Yamaguti Univ., 16: 607 (1965).

252. G. rubi Sawada, Report of the Department of

Agriculture, Government Research Institute of Formosa

85: 31 (1943)

253. G. rugosa T.S. Ramakr., Sriniv. \& Sundaram,

Proc. Indian Acad. Sci., Pl. Sci. 37(3): 85 (1953)

254. G. salicis (Fuckel) Syd. ex Kirschst., Annls mycol. 34(4/5): 318 (1936)

255. G. salicina Hara

(Ref. Index fungorum)

256. G. sansevieriae Punith., Mycol. Pap. 136: 45 (1974)

IMI, U.K

257. G. sarcomphali Gonz. Frag. \& Cif., Rep. Dominic. Est. MA, Spain

Agron. Haina, Ser. B, Bot. Bull. 8: 20, fig. 9 (1927)

TNS, TFM, Not

NBRC and examined

YAM, Japan

BPI, U.S.A

Examined

MA, Spain

Examined

Not

examined

Examined

Examined

YAM, Japan

Examined

NTU, Taiwan

$\mathrm{AMH}$, India and

F, U.S.A

TNS,

NBRC

YAM, Japan

TFM,
and

\section{Examined}

Not

examined

258. G. sawadae Tak. Kobay., in Kobayashi \& Sasaki, Trans. NBRC, TNS, Not Mycol. Soc. Japan 16: 234 (1975) K, U.K
TFM and YAM, examined Japan
No

communication or specimen received from herbarium.

No

communication or specimen received from herbarium. Specimen in poor condition.

No

communication or specimen received from herbarium.

No

communication or specimen received from herbarium.

No

communication or specimen received from herbaria.

Specimen in poor condition

Specimen in poor condition

No

communication or specimen received from herbaria

This is a

Phyllochora species (tarspot)

(Fig. 17t-z $\mathrm{z}^{3}$ )

This is an unknown ascomycete (Fig. $17 z^{4}-z^{10}$ )

Specimen in poor condition.

Specimen in poor condition.

No

communication or specimen received from herbaria.

Specimen in poor condition.

No

communication or specimen received from herbaria.

Specimen in poor condition.

No

communication or specimen received from herbarium.

No

communication or specimen received from herbaria. 
259. G. scabiosae (Lambotte \& Fautrey) Arx \& E. Müll., Bei BPI, U.S.A Kryptfl. Schweiz 11(no. 1): 53 (1954)

260. G. scirpicola Grab., Bull. Soc. mycol. Fr. 33: 76 (1917)

261. G. seriata Bäumler, Verh. Ver. Nat., Heilk. Pressb., N.F. 23: 26 (1902)

262. G. serratullae (Petr.) E. Müll., Sydowia Beih. 1: 213 (1957)

263. G. sibirica Lavrov, in Trud. Tomsk. Gos. Univ.

Kuibysheva, Ser. Boil., 110(4): 46 (1951)

264. G. singularis (Magnus) Arx \& E. Müll., in Müller \& von Arx, Beitr. Kryptfl. Schweiz 11(no. 2): 25 (1962)

265. G. smilacicola Sawada, Special Publication College of Agriculture, National Taiwan University 8: 59: (1959)

266. G. smilacicola Sawada ex W.Y. Li \& W.Y. Zhuang, in Li \& Zhuang, Mycotaxon 100: 219 (2007)

267. G. sojae Sawada, Special Publication College of Agriculture, National Taiwan University 8: 60 (1959)

268. G. spinicola (Sacc.) Lindau, in Engler \& Prantl, Nat. Pflanzenfam., Teil. I (Leipzig) 1(1): 423 (1897) 269. G. stromatica (Fuckel) Petr., Annls mycol. 21(3/4): 269 (1923)

270. G. sudetica Petr. Annls mycol. 19(1/2): 104 (1921)

271. G. sydowiana Trotter, in Saccardo, Syll. fung. (Abellini) 24(2): 788 (1928)

272. G. synedrellae Syd., Ann. Mycol. 14: 360 (1916)

273. G. theae (Racib.) G. E. Bernard, Bull. Dept. Agric. Indes Netherland 6: 26 (1907)

274. G. tilakii R. Rao \& S.B. Kale, Mycopath. Mycol. appl. 27(1-2): 114 (1965)

275. G. tofieldiae (Lambotte \& Fautrey) Arx \& E. Müll., Beitr. Kryptfl. Schweiz 11(1): 59 (1954)

276. G. traversoana Gonz. Frag., Trab. Mus. Nac.

Cienc. Nat., Ser. Bot.: 73 (1916)
B, Germany

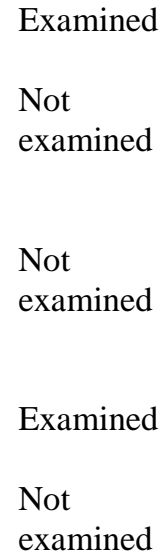

ZT, Switzerland

Not

examined

TNS, Japan

Examined

HMAS, China

Not

examined

NTU, Taiwan

PAD, Italy

MA, Spain

MA, Spain

PAD, Italy

F, U.S.A

KRA, Poland

AMH, India

SIENA, Italy

MA, Spain
Examined

Not

examined

Examined

Examined

Not

examined

Examined
Specimen in poor condition.

No

communication or specimen received from herbarium.

No

communication or specimen received from herbarium. Specimen in poor condition

No

communication or specimen received from herbarium. No

communication or specimen received from herbarium This is a sexual state of Colletotrichum sp. (Glomerella sp.)

(Fig. 18a-i). Currently invalid name (Index Fungorum). No communication or specimen received from herbarium.

This is sexual state of Colletotrichum (Glomerella sp.) (Fig. 18j-p). Specimen is not holotype. Specimen is not holotype.

This is an unknown bitunicate ascomycete (Fig. 18q-w).

No

communication or specimen received from herbarium. Specimen in poor condition.

Specimen in poor condition.

No

communication or specimen received from herbarium. Poor condition of the specimen. The specimen contained an unknown 
277. G. trichosanthis Katsuki, J. Jap. Bot. 31(12): 370 (1956)

278. G. tumefaciens Cribb, Pap. Dept. Bot. (formerly Biol.) I BRIP, Australia (1954)

279. G. tunetana (Pat.) Sacc., Syll. fung. (Abellini) 24(2): 79 PH, U.S.A (1928)

280. G. ulmariae Miura, Petr. List. Suppl.: 160 (1928)

281. G. ulvae M. Reed, University of Calif. Publ. Bot. 1: 160 (1902)

282. G. umbelliferarum (Rabenh.) Petr., Annls mycol. 19(1/2): 106 (1921)

283. G. veneta (Sacc. \& Speg.) Traverso, Fl. Ital. Crypt. 2:392 (1906)

284. G. valesiaca Arx \& Müll., Beitr. Kryptfl. Schweiz 11(no. 1): 58 (1954)

285. G. veronicae (Rostr.) P. Larsen in Rosenvinge \& Warming, in Botany of Iceland 2(3): 832, 9 (Fungi of Island) (1932)

286. G. verrucicola (Wedd.)

Keissl., Rabenh. Krypt.-Fl., Edn 2 (Leipzig) 8: 341 (1930)

287. G. verrucicola f.

olivieri (Vouaux) Keissl., Rabenh. Krypt.-Fl., Edn 2

(Leipzig) 8: 342 (1930)

288. G. verrucicola f.

verrucicola (Wedd.) Keissl., Rabenh. Krypt.-Fl., Edn 2

(Leipzig) 8: 341 (1930)

289. G. xanthosomatis Cif. \& Gonz. Frag., Boln de la Real

Soc. Españ. Hist. Nat., Madrid 26: 493 (1926)

290. G. xylostei Reusser,

Phytopathologische Zeitschrift 51: 223 (1964)
NBRC, TFM,

TNS and YAM.

Not

examined

Not

examined

Examined

TNS,

Japan

TFM, Not

examined

PAD, Italy

Not

examined

B (Germany), K (U.K)

Not

examined

PAV, Italy

W, Austria

C, Denmark

PC, France

Not

examined

K (U.K),

PC (France),

W (Austria)

PC, France

Not

examined

BPI, U.S.A

ETH,

Switzerland ascomycete.

No

communication or specimen received from herbaria.

No

communication or specimen received from herbarium.

The specimen contained a

Pleospora

species.

No

communication or specimen received from herbaria.

No

communication or specimen received from herbarium.

No

communication or specimen received from herbaria.

Examined Specimen in poor condition.

Not No

Examined communication or specimen received from herbarium. Specimen in poor condition.

No

communication or specimen received from herbarium.

No

communication or specimen received from herbaria.

No

communication or specimen received from herbarium.

Examined Specimen in poor condition.

Examined The specimen contained a Venturia-like species (Fig. 18x$z^{5}$ ).

Abbreviation: AA: Herbarium Institute of Botany and Phytointroduction, Ministry of Science, Academy of Sciences, 44 Temirajzev Street, Alma-Ata 480070, Kazakhstan; AMH: Herbarium, Mycology and Plant Pathology Department, Plant Sciences Division, Agharkar Research Institute, G. G. Agarkar Road, Pune 411 004, Maharashtra, India; B: Botanischer Garten und Botanisches Museum, Berlin, Germany; BISH: Bernice P. Bishop Museum, Honolulu, Hawaii; BM: Herbarium Department of Botany, The Natural History Museum, England U.K; BO: Herbarium Bogoriense, Cibinong, Indonesia, BPI: US National Fungus Collection (Beltsville, Md, USA); BR: Jardin botanique national national de Belgique, Belgium; BRIP: Plant Pathology Herbarium. Department of Primary Industries, Queensland. Australia; BUCM: Mycology Herbarium, Centre of Ecology, Taxonomy and Nature Conservation, Institute of Biology Bucharest, Romanian Academy, 296 Splaiul Independentei, P.O. Box 56-53, 060031 Bucuresti, Romania; C: Natural 
History Museum of Denmark. Denmark, Coppenhagen; CBS: CBS-KNAW Fungal Biodiversity Centre, Uppsalalaan 8, 3584 CT, Utrecht, The Netherlands; DAOM: National Mycological Herbarium, Biosystematics Research Institute, Canada Agriculture, Central Experimental Farm, Ottawa, Ontario, Canada; DAR: New South Wales department of Agriculture, Australia; ETH: National Herbarium. Biology department. Science Faculty. Addis Ababa. Ethiopia; F: Herbarium Botany Department, Field Museum of Natural History, 1400 South Lake Shore Drive Chicago, Illinois 60605-2496, U.S.A; FH: Farlow Herbarium and reference library, Harvard University of Massachusetts, U.S.A; G: Conservatoire et Jardin Botanique, Switzerland; HBG: Herbarium Biozentrum Klein-Flottbek, Ohnhorststrasse 18, D22609 Hamburg, Germany; HMAS: Chinese Academy of Sciences, Beijing, China; ILL: Department of Botany, University of Illinois, Urbana, Illinois, U.S.A; IMI: Commomwealth Mycological Institute, Surrey, England; K: Royal Botanic Garden Herbarium, Kew, U.K; L: National Herbarium of the Netherlands, Leiden; KM: Herbarium Krkonošské museum, Správa Krkonošského národního parku, Dobrovského 354311 Vrchlabí, Czech Republic; KRA: Herbarium Institute of Botany, Jagiellonian University 31 Kopernika Street, PL-31-502 Kraków, Poland; LE: Herbarium Russian Academy of Sciences, V. L. Komarov Botanical Institute, Prof. Popov Street 2, Saint Petersburg 197376, Russia; LISE: Herbário Fitosistemática e Geobotânica, Estação Agronómica Nacional, Portugal; LPS: Instituto de Botánica C. Spegazzini, La Plata, Argentina; MA: Herbario Real Jardín Botánico, Plaza de Murillo 2 , 28014 Madrid, Spain; MFU: Mae Fah Luang Herbarium, Chiang Rai, Thailand; MW: Herbarium Biological Faculty, Moscow State University, Vorobjovy Gory, Moscow 119991, Russia; NBRC: Culture Collection Division, NITE Biological Resource Center, National Institute of Technology and Evaluation, 2-5-8 Kazusakamatari, Kisarazu-shi, Chiba 292-0818, Japan; NTU: The Herbarium of National Taiwan University, Taiwan; P: Herbier National de Paris, Département de Systématique et Evolution, Phanérogamie, Muséum National d'Histoire Naturelle, 16 rue Buffon, F75005 Paris, France; PAD: Università degli Studi di Padova, Italy; PAV: Erbario Dipartimento de Ecologia del Territorio, Università di Pavia, Via S. Epifanio 14, I-27100 Pavia, Italy; PARMA: Erbario Istituto ed Orto Botanico, Università degli Studi di Parma, Via Farini 90I-43100 Parma, Italy; PC: VHerbier Cryptogamique, Dépt. Systématique et Évolution, Muséum National d'Histoire Naturelle, France; PDD: New Zealand Fungal Herbarium, Landcare Research, Auckland, New Zealand; PREM: National Collection of Fungi of the Republic of South Africa (Pretoria, South Africa); RB: Herbário Instituto de Pesquisas, Jardim Botânico do Rio de Janeiro, 22460-030 Rio de Janeiro, Rio de Janeiro Brazil; S: Herbarium Swedish Museum of Natural History, P.O. Box 50007, Svante Arrhenius väg 7, S-104 05 Stockholm, Sweden; SIENA: Herbarium Universitatis Senensis, Dipartimento di Scienze Ambientali "G. Sarfatti", Sezione Museo Botanico, Università di Siena, Via Pier Andrea Mattioli 4, 53100 Siena, Italy; STR: Herbier Institut de Botanique, 28 rue Goethe, F-67083 Strasbourg Cedex, France; TNS: Herbarium Department of Botany. National Museum of Nature and Science, Tsukuba, Japan; TFM: Forestry and Forest Products Reseach Institute, Japan; UC: University Herbarium, University of California, 1001 Valley Life Sciences Building \#2465, Berkeley, California 94720-2465, U.S.A; URM: Herbário Departamento de Micologia, Centro de Ciências Biológicas, Universidade Federal de Pernambuco 50670-420 Recife, Pernambuco, Brazil; VER: Erbario Sezione di Botanica, Museo Civico di Storia Naturale, Corso Cavour 11, I-37121 Verona, Italy; YAM: Herbarium Plant Pathology Department. Faculty of Agriculture. Yamaguchi University. Yamaguchi-shi, Yamaguchi, Japan; VIC: Herbário Departamento de Biologia Vegetal Universidade Federal de Viçosa, Brazil; W: Naturhistorisches Museum, Wien (Vienna, Austria); YAM: Herbarium Plant Pathology, Faculty of Agriculture, Yamaguchi University, Japan; ZT: University of Zurich \& ETH Herbarium of Switzerlands.

\section{Discussion}

A large number of species have been described in the genus Guignardia that has its asexual morph in Phyllosticta. An effort was made to re-visit the genus Guignardia by obtaining on loan and examining the type material of the species deposited in various herbaria. In this paper, of the reviewed 304 species, in Section I, forteen species are transferred away from Guignardia and grouped in two other families (Botryosphaeriaceae and Hyponectriaceae) and four genera (Botryosphaeriaceae, Vestergrenia, Neodeightonia, Hyponectria). In section II, 107 treated as doubtful; identity of 183 species specimens could not be confirmed with certainty in view of nonreceipt of holotypes from the herbaria where these are originally deposited and poor condition of those received specimens (Table 2).

Though we did not get specimens for 183 species, interesting responses were received from the herbaria world around. A few reasonings are considered here.

1. Some specimens are untraceable or missing in the herbaria because of destructions during World War II, such as B.

2. A few packets received were without the appropriate fungal type materials, as in the case of MA.

3. Some specimens were loaned elsewhere, such as G. aegyptiaca in G (Herbarium Conservatoire et Jardin botaniques de la Ville de Genève) and could not be obtained during the period of this study.

4. Herbaria such as HMAS (China), AMH (India) did not respond our requests. 
5. Some herbaria sent some other collections and not the holotypes that we were looking for, such as MA, with Guignardia oxyriae, G. buxi and others.

6. Many herbaria specimens contained fungi other than Guignardia.

7. In a few instances, the specimens that are listed in Index Fungorum did not exist and taxonomy of those fungi has become nomen invalid (invalid name).

About 107 taxa remained doubtful because the specimens were as under:

1. Limited number of ascomata in the specimens examined.

2. Some holotype specimen packets contained only mounted slides, which unfortunately were not in good condition.

3. Some dried leaves or stem specimens with holotype were in such poor conditions that all needful characters of the fungi could not be asertained; in such cases, the taxa are treated as doubtful.

4. In species with asexual states and so far not linked to sexual states, it is unclear if the taxa are valid. Holotype specimen of Guignardia himalayensis from DAOM and ZT are Kabatia sp. Sivanesan (1984) stated that Kabatia is an anamorph of Discosphaerina whereas Kirk et al. (2008) mentioned this species in Dothioraceae and linked to Discoshaerina. Lumbsch (2010) and Wijayawardene et al. (2011) referred Dischospahaerina in Hyponectriaceae. Based on these varied and conflicting observations, the position of Kabatia remained uncertain. Hyponectriaceae is a family with unitunicate asci. Sivanesan's (1984) suggestion that Kabatia is synonym to Discosphaerina is also now not tenable.

Fresh collections, cultures and molecular sequencing are needed to clarify the phylogeny and taxonomy of all those doubtful species and many cryptic species.

\section{Acknowledgements}

This study was funded in part by the Thailand Research Fund (grant DBG5380011). The Directors and Curators of the International Herbaria BPI, C, DAR, ETH, F, FH, ILL, IMI, KM, MA, NTU, PAV, PH, PREM, TNS, TUR, VIC, W, YAM, and ZT are profoundly thanked for loaning specimens, including the holotype. Mushroom Research Foundation is thanked for a PhD scholarship. Hong Kong University, Hong Kong and the School of Science, Mae Fah Luang University, Thailand and CBS, The Netherlands are thanks for laboratory facilities. Dr Kevin D. Hyde thank you for valuable comments on manuscript. Dr Pedro W Crous is thanked for partial funding of this research.

\section{References}

Aa HA van der 1973 - Studies in Phyllosticta I. Studies in Mycology 5, 1-110.

Aa HA Van der, Vanev S. 2002 - A Revision of the species described in Phyllosticta. Centraalbureau voor Schimmelcultures, Utrecht, The Netherlands. 1-49.

Arx JA von, Müller E. 1954 - Die Gattungen der Amerospores Pyrenomyceten. Beitr Kryptogamenflora Schweiz 11, 1-434.

Barr ME. 1977 - Magnaporthe, Telimenella, and Hyponectria (Physosporellaceae). Mycologia 69, 952-966.

Chattaoui M, Rhouma A, Msallem M. 2012 - First Report of Botryosphaeria obtusa as Causal Agent of Olive Tree Branch Dieback in Tunisia. Plant Disease 96 (6), 905.

Hyde KD. 1995 - The genera of Guignardia from Palm XX. Sydowia 47, 180-198.

Hyde KD et al. 2013 - Families of Dothideomycetes. Fungal Diversity. Paper in progress.

Index Fungorum-http://www.indexfungorum.org/; accessed December - 2012.

Kirk PM, Cannon PF, Minter DW, Stalpers JA. 2008 - Dictionary of Fungi. Ten Edition. CAB International. Wallingford. Oxon. OX 10 8DE. U.K., 771.

Liu JK, Phookamsak R, Doillom M, Wikee S, Li YM, Ariyawansa H, Boonmee S, Chomnunti P, Dai DQ, Bhat JD, Romero AI, Zhuang WY, Monkai J, Jones EBG, Chukeatirote E, Ko Ko TW, Zhao YC, Wang Y, Hyde KD. 2012 - Towards a natural classification of Botryosphaeriales. Fungal Diversity 57, 149-210. 
Liu JK, Phookamsak R, Jones EBG, Zhang Y, Ko-Ko TW, Hu HL, Boonmee S, Doilom M, Chukeatirote E, Bahkali A.H, Wang Y, Hyde K.D. 2011 - Astrosphaeriella is polyphyletic with species in Fissuroma gen. nov., and Neoastrosphaeriella gen. nov., Fungal Diversity $51,135-54$.

Lumbsch HT, Huhndorf SM 2010 - Outline of Ascomycota - 2009; Notes on ascomycete systematics. Nos. 4751-5113. Myconet 14, 1-69.

Macedo DM, Barreto RW 2008 - First record of Botryosphaeria ribis associated with leaf spots on Magnolia aff. candollei in Brazil. Brazilian Journal of Microbiology 39, 321-324.

Monkai J, Liu JK, Boonmee S, Chomnunti P, Chukeatirote E, Jones EBG, Wang Y, Hyde KD. 2013 - Planistromellaceae (Botryosphaeriales). Cryptogamie Mycologie 34, 11.

Motohashi K, Araki I, Nakashima C. 2008 - Four new species of Phyllosticta, salora from Japan. Mycoscience 49, 138-146.

Motohashi K, Kobayashi T, Furukawa T, Ono Y. 2010 - Notes on some plant-inhabiting fungi collected from the Nansei Island (2). Mycoscience 51, 93 - 97.

Okane I, Lumyong S, Nakagiri A, Ito T. 2003 - Extensive host range of an endophytic fungus, Guignardia endophyllicola (anamorph: Phyllosticta capitalensis). Mycoscience 44, 353363.

Okane I, Nakagiri A, Ito T. 2001 - Identity of Guignardia sp. inhabiting ericaceous plants. Can. J. Bot., 79, $101-109$.

Punithalingam E. 1974 - Studies on sphaeropsidales in culture II. Mycological Papers 136, 1-63.

Sivanesan A. 1984 - The Bitunicate Ascomycetes and their anamorphs. J. Cramer, Vaduz., 701.

Slippers B, Crous PW, Denman S, Coutinho TA, Wingfield BD, Wingfield MJ. 2004 - Combined multiple gene genealogies and phenotypic characters differentiate several species previously identified as Botryosphaeria dothidea. Mycologia 96, 83-101.

Wijayawardene DNN, McKenzie EHC, Hyde KD. 2012 - Towards incorporating anamorphic fungi in a natural classification-checklist and notes for 2011. Mycosphere 3(2), 157-228, Doi 10.5943/mycosphere/3/2/5/.

Wulandari NF, Bhat DJ, To-anun C 2013 - A modern account of the genus Phyllosticta. Plant Pathology \& Quarantine 3(2), 145-159, doi 10.5943/ppq/3/2/4

Wulandari NF, To-anun C, Crous PW, Hyde KD. 2010 - "Guignardia/Phyllosticta from northern Thailand", Proceedings of the International Conference of Association Tropical Biodiversity Conservation (ATBC), Bali, Indonesia, 149.

Wulandari NF, To-anun C, Hyde KD, Duong LM, Gruyter J de et al. 2009 - Phyllosticta citriasiana sp. nov., the cause of Citrus tan spot of Citrus maxima in Asia. Fungal Diversity 34, 23-39.

Yamamoto W. 1961 - Species of the Genera of Glomerella and Guignardia with special reference to their imperfect stages. Sci. Rep. Hyogo Univ. Agric. 5, 112. 


\section{Appendix I}

In those doubtful species referred in Section II, a few specimens had ascomata or conidiomata. Majority of these were in poor condition and the taxonomy could not be ascertained. Nevertheless, wherever possible, morphology was studied and whatever fungi observed are illustrated and parked here in Fig. 11-18 with legends. Notes are given in the last column of Table 2 under inference.
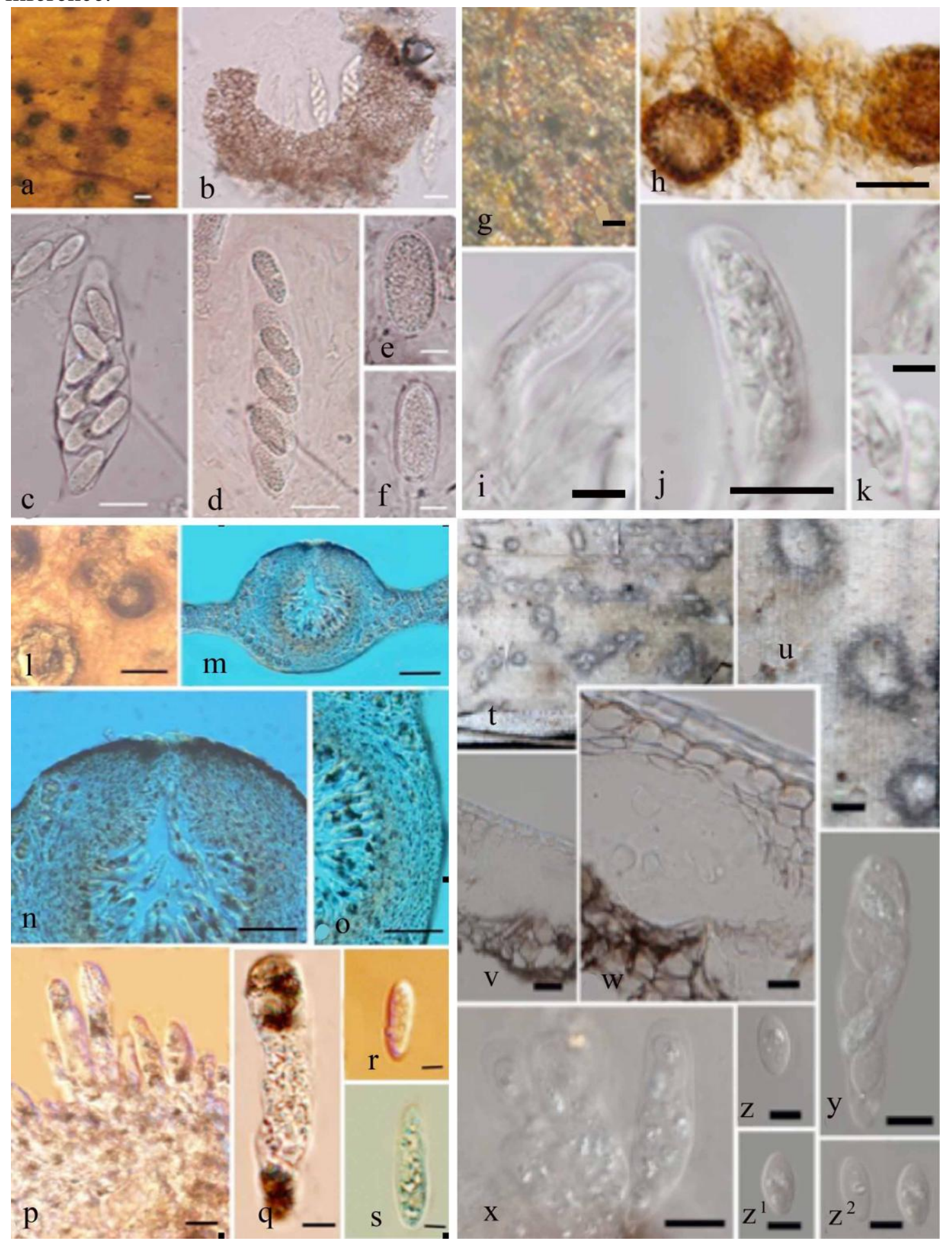

Figs 11a-f - Colletotrichum sp. (from holotype of Guignardia adeana) - 11g-k - Unknown ascomycete (from holotype of G. agerati) - 11l-s - Mastodia tessalata (from holotype of $G$. alaskana) - 11t-z - Botryosphaeria sp. (from holotype of G. arengae Rehm). 


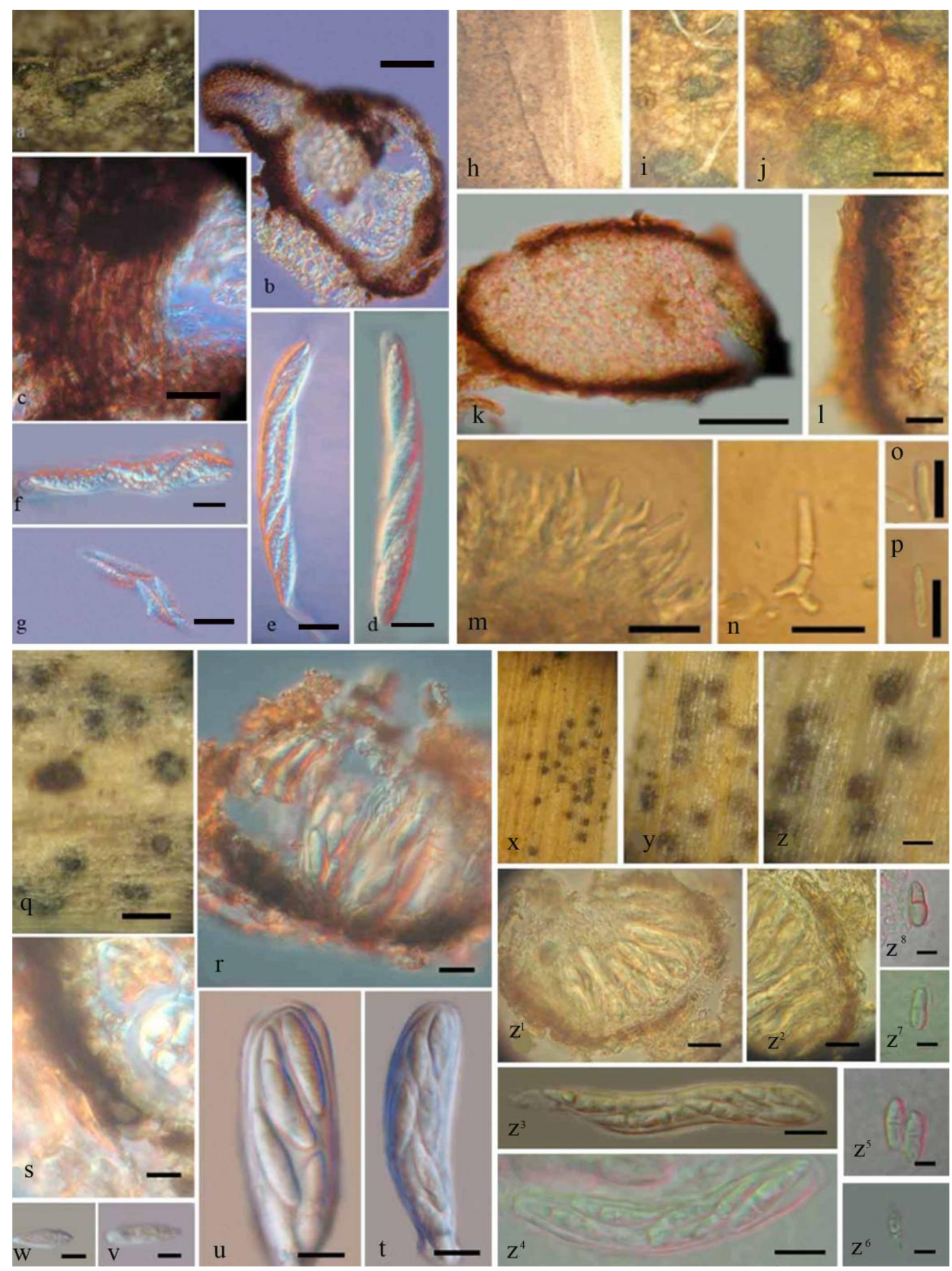

Figs 12a-g - Unknown ascomycete (from holotype of Guignardia bambusina Rehm) - 12h-p Unknown coelomycete (from holotype of G. biennis) - 12q-w - Botryosphaeria-like (from holotype of G. boltoniae) - 12x-z $\mathbf{z}^{\mathbf{8}}-$ Mycosphaerella-like (from holotype of G. caricis). 


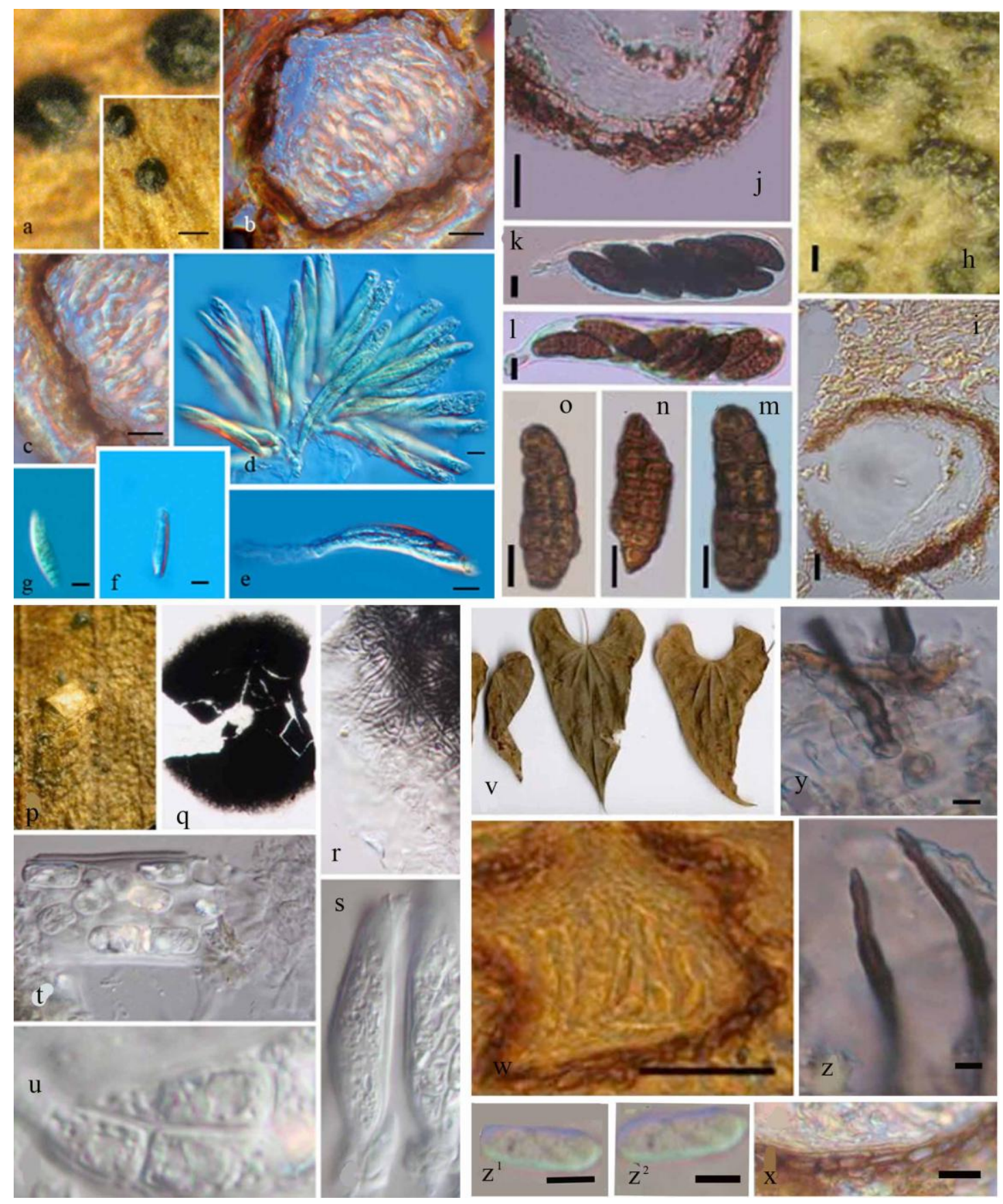

Figs 13a-g - Unknown ascomycetes (from holotype of Guignardia clusiae) - 13h-o - Pleosporalike (from holotype of G. cytisi) - 13p-u - Micropeltis-like (from holotype of G. dieffenbachiae

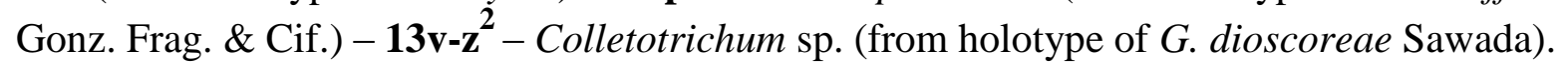




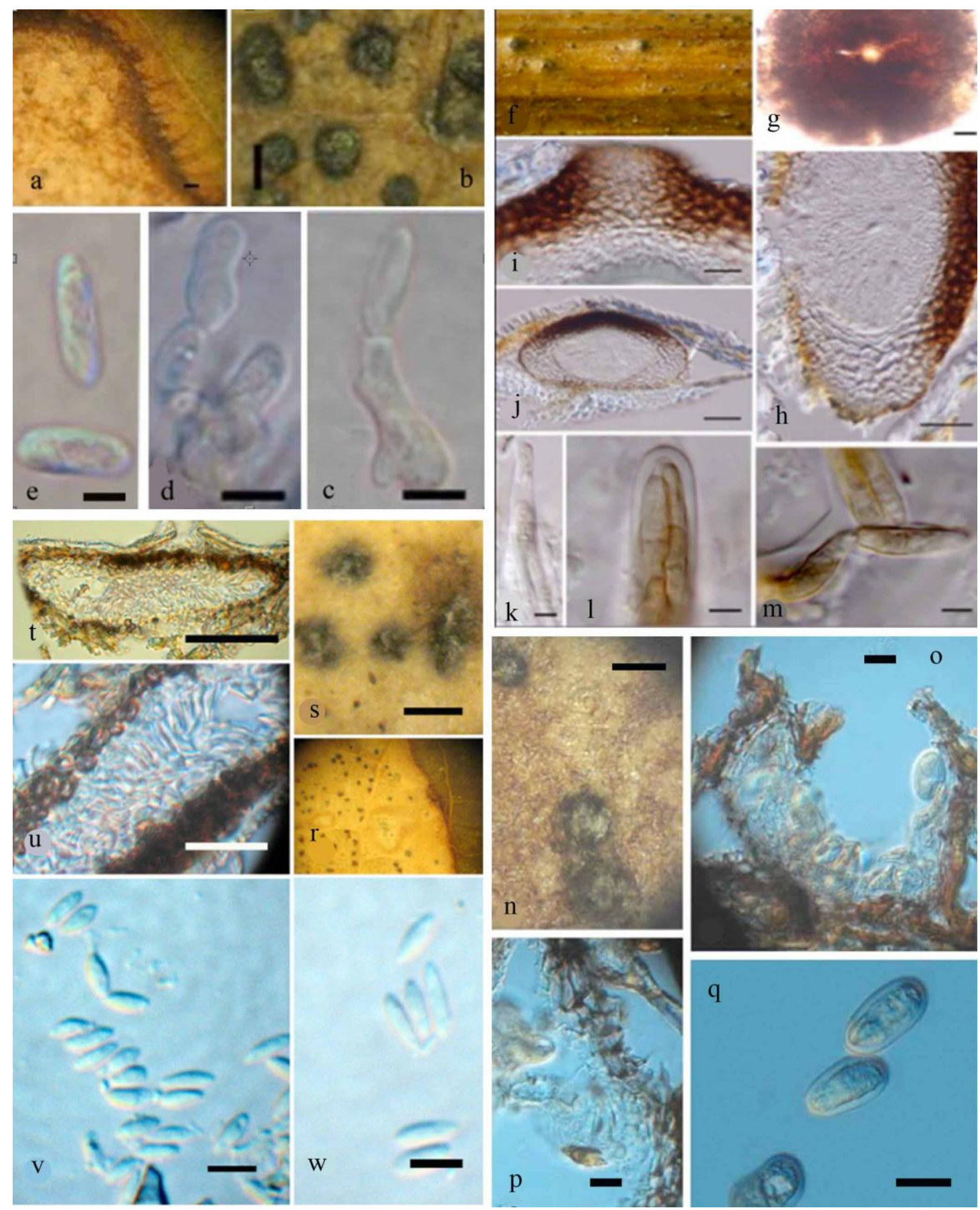

Figs 14a-w - Colletotrichum sp. (from holotype of Guignardia fatsiae) - 14f-m - Unknown ascomycete (from holotype of G. franconicae) - 14n-q - Unknown coelomycte (from holotype of G. fuscocoricae) - 14r-w - Phomopsis-like (from holotype of G. heveae Syd. \& P. Syd.). 


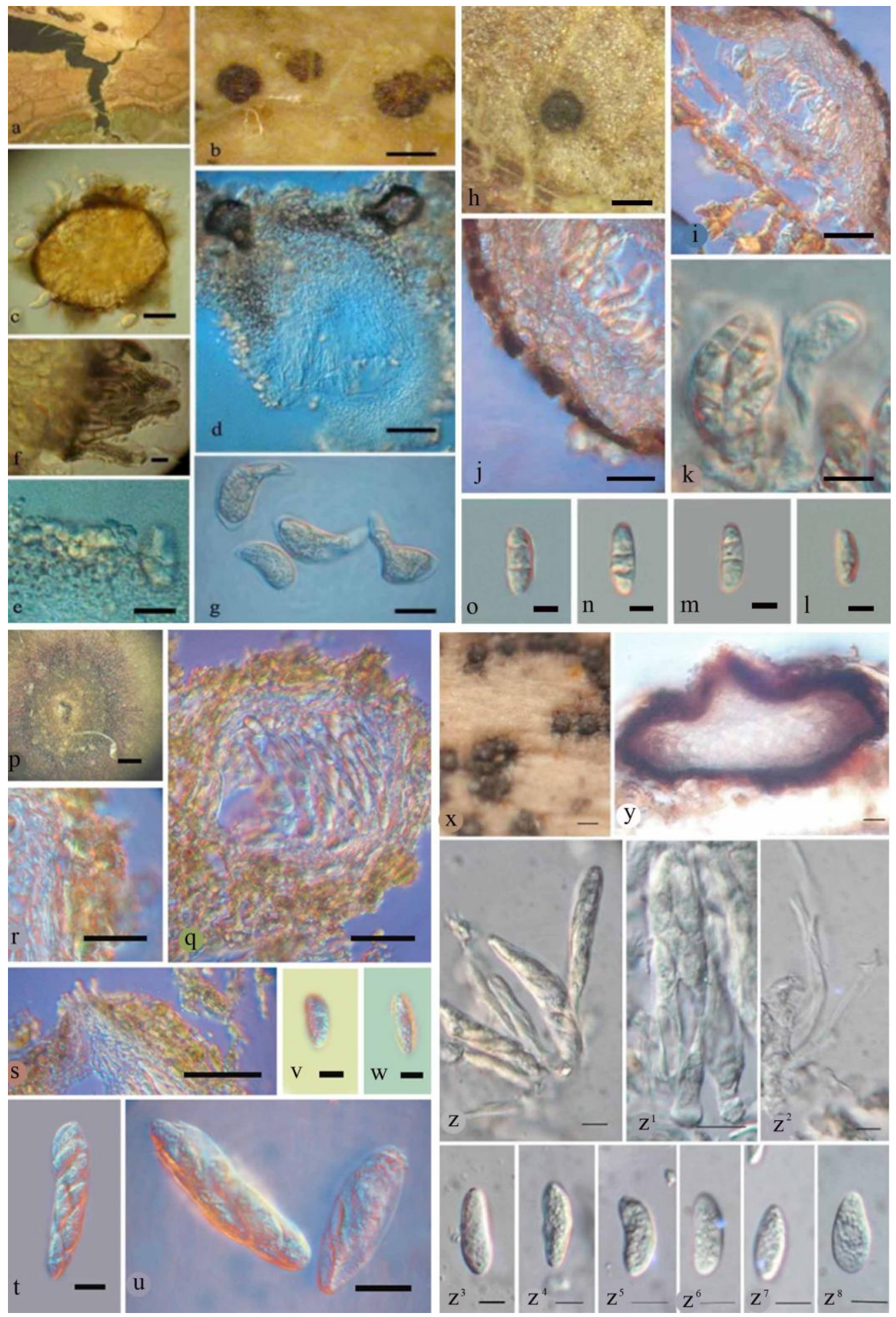

Figs 15a-g - Kabatia sp. (from holotype of Guignardia himalayensis) - 15h-o - Irenopsis ingae (from holotype of $G$. ingae) - 15p-w - Unknown ascomycete (from holotype of G. jussiaeae) - 15x$\mathbf{z}^{\mathbf{8}}$-Colletotrichum sp. (from holotype of G. manihoticola). 

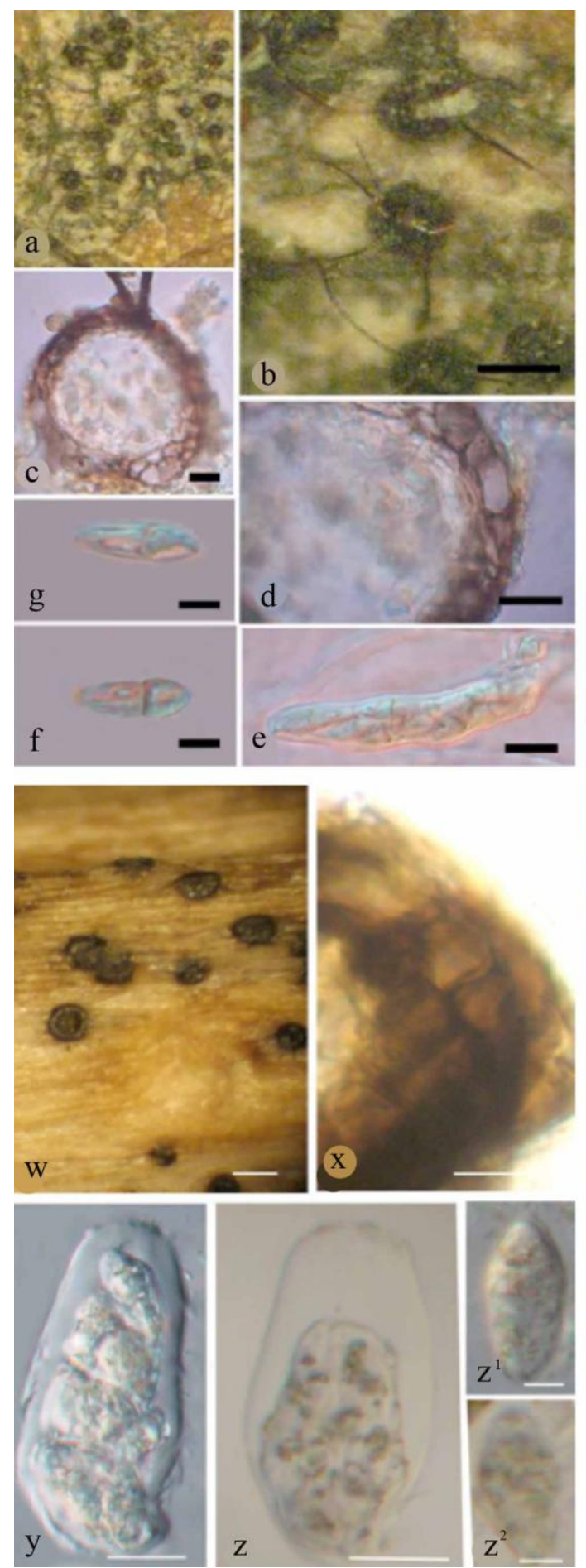
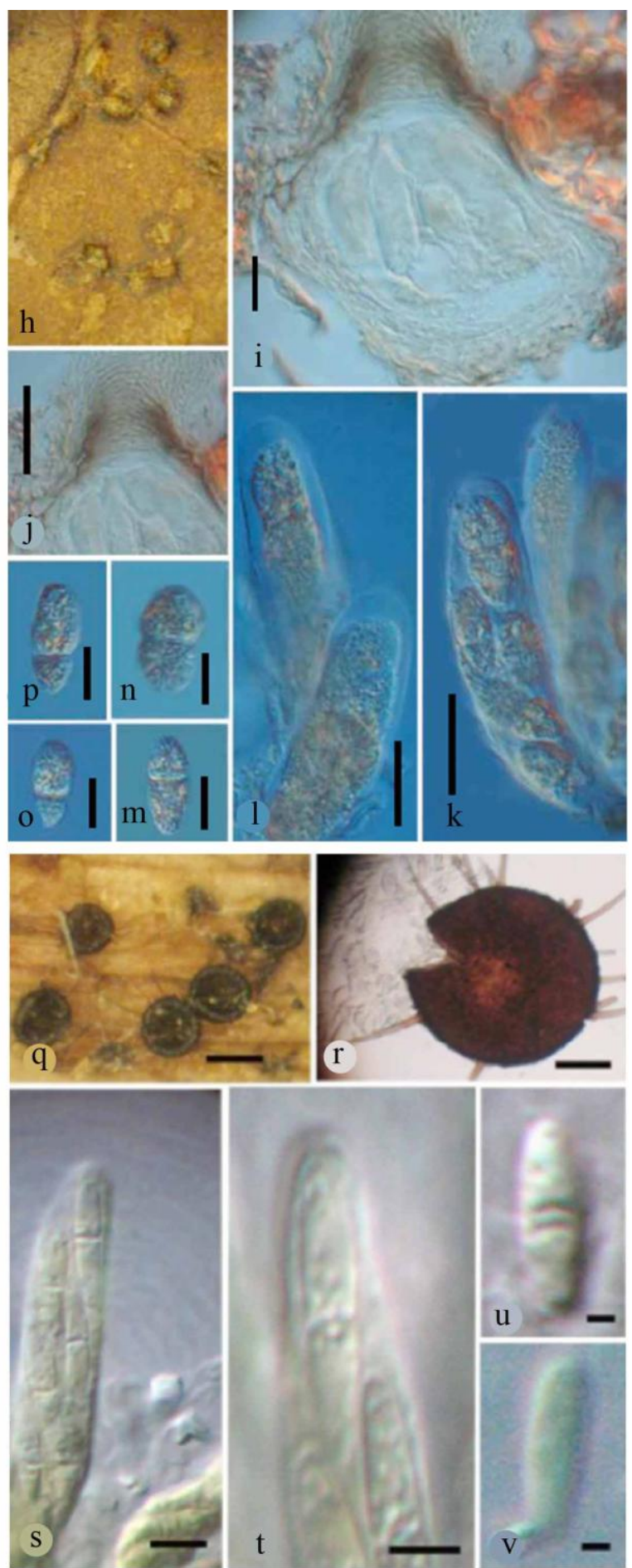

Figs 16a-g - Kabatia sp. (from holotype of Guignardia mirabilis) - 16h-p - Dothidea-like (from holotype of $G$. nectandrae) - 16q-v - Unknown ascomycete (from holotype of G. oxyriae) - 16w-z $\mathbf{z}^{2}$ - Botryosphaeria-like (from holotype of G. pegani). 

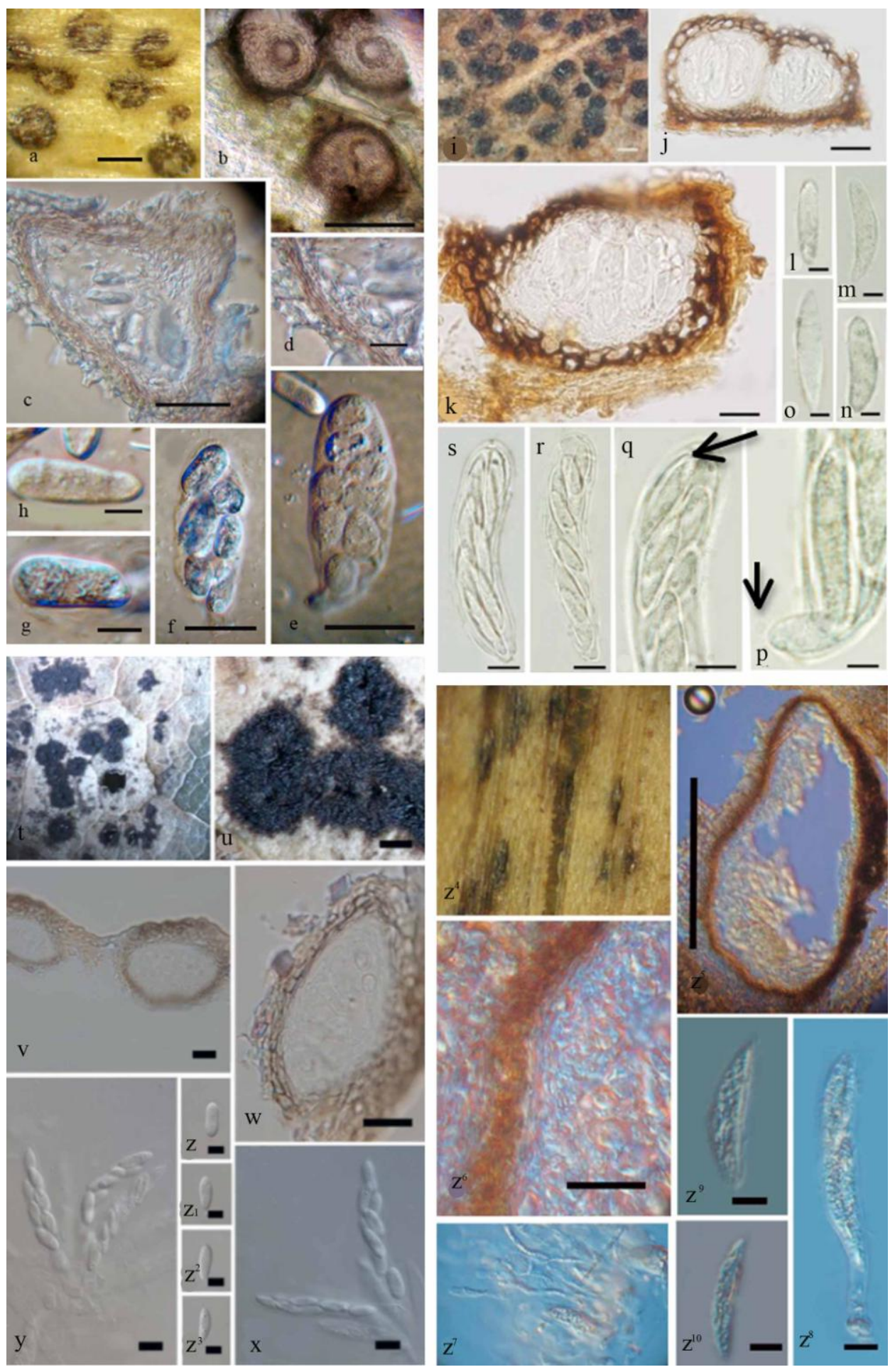

Figs 17a-h - Colletotrichum sp. (from holotype of Guignardia plectroniae) - 17i-s Physalospora-like (from holotype of G. populi) - 17t-z $\mathbf{3}^{\mathbf{3}}$ - Phyllachora sp. (from holotype of $G$. rhytismophila) - $\mathbf{1 7 z}^{\mathbf{4}}-\mathbf{z}^{\mathbf{1 0}}$ - Unknown ascomycete (from holotype of G.rhynchosporae). 

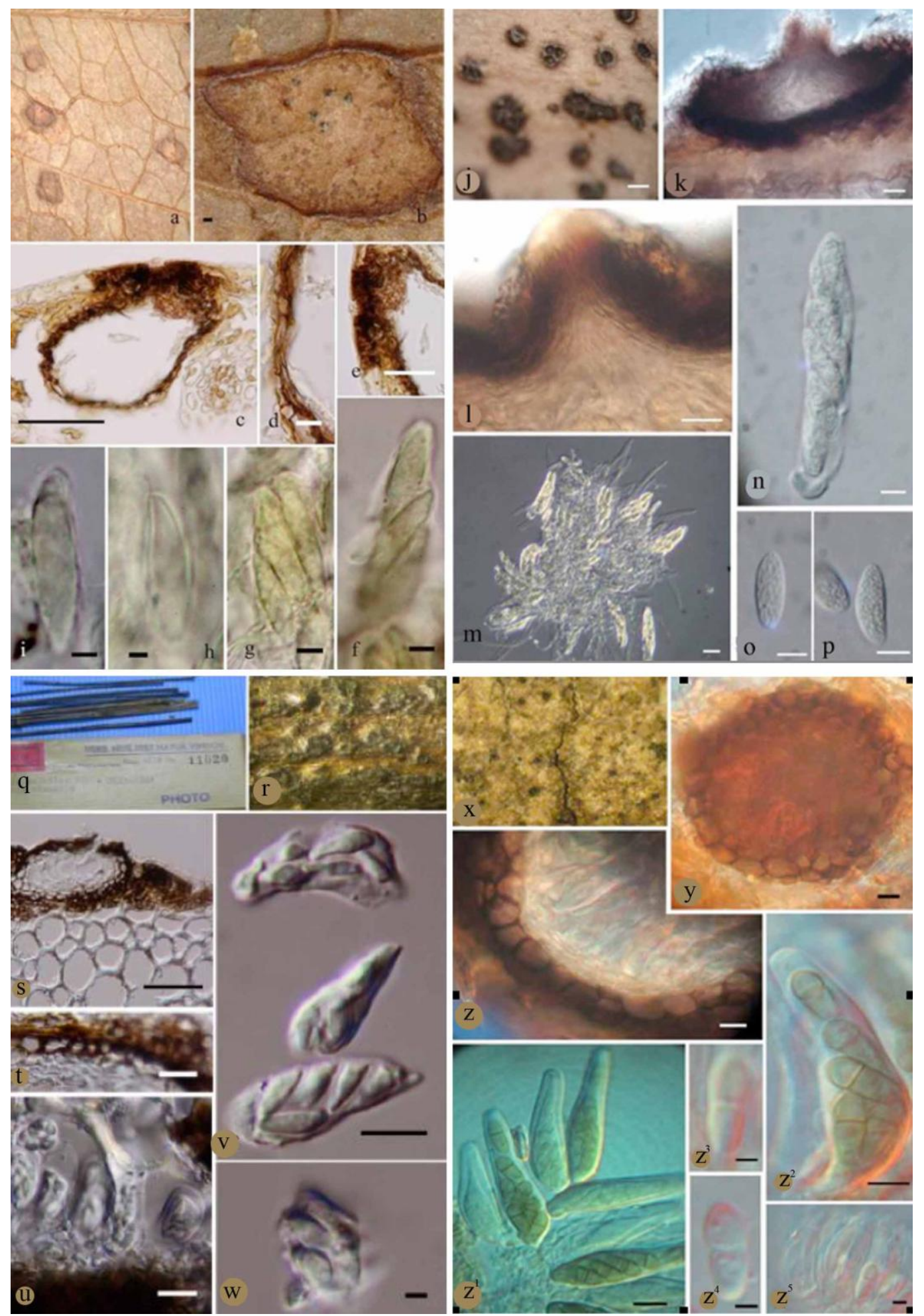

Figs 18a-i - Colletotrichum sp. (from holotype of Guignardia smilacicola) - 18j-p Colletotrichum sp. (from holotype of G. sojae). 18q-w - Unknown bitunicate ascomycete (from holotype of Guignardia sudetica). 18x-z - Venturia-like (from holotype of Guignardia xylostei). 